\title{
Isogeometric Analysis of Geometric Partial Differential Equations
}

\author{
Andrea Bartezzaghi ${ }^{\mathrm{a}, *}$, Luca Dedè ${ }^{\mathrm{a}}$, Alfio Quarteroni ${ }^{\mathrm{a}, \mathrm{b}}$ \\ ${ }^{a}$ CMCS - Chair of Modeling and Scientific Computing, MATHICSE - Mathematics Institute of Computational \\ Science and Engineering, EPFL - École Polytechnique Fédérale de Lausanne, \\ Station 8, Lausanne, CH-1015, Switzerland \\ ${ }^{b}$ MOX - Modeling and Scientific Computing, Department of Mathematics, Politecnico di Milano, \\ Piazza L. da Vinci 32, Milano, 20133, Italy (on leave)
}

\begin{abstract}
We consider the numerical approximation of geometric Partial Differential Equations (PDEs) defined on surfaces in the 3D space. In particular, we focus on the geometric PDEs deriving from the minimization of an energy functional by $L^{2}$-gradient flow. We analyze two energy functionals: the area, which leads to the mean curvature flow, a nonlinear second order PDE, and the Willmore energy, leading to the Willmore flow, a nonlinear fourth order PDE. We consider surfaces represented by single-patch NURBS and discretize the PDEs by means of NURBS-based Isogeometric Analysis in the framework of the Galerkin method. To approximate the high order geometric PDEs we use high order continuous NURBS basis functions. Instead, for the time discretization of the nonlinear geometric PDEs, we use Backward Differentiation Formulas (BDF) with extrapolation of the geometric quantities involved in the weak formulation of the problem; in this manner, we solve a linear problem at each time step. We report numerical results concerning the mean curvature and Willmore flows on different geometries of interest and we show the accuracy and efficiency of the proposed approximation scheme.
\end{abstract}

Keywords: Geometric Partial Differential Equation, Surface, High Order, Isogeometric Analysis, Mean Curvature Flow, Willmore Flow

\section{Introduction}

Geometric Partial Differential Equations (PDEs) describe the evolution of the geometrical domain in which these equations are set [1]; such problems are usually defined on surfaces in 3D and the surface itself represents the unknown of the geometric PDE. The computational domain evolves in time, or pseudo-time, according to geometric quantities of interest, such as the curvature of the

\footnotetext{
${ }^{*}$ Corresponding author.

Email address: andrea.bartezzaghi@epfl.ch (Andrea Bartezzaghi)
} 
surface, towards the (local) minimization of the associated energy functional. Problems of this kind arise in different applications; examples are material Science, where the crystalline structure or the geometric properties of the materials can be described via mathematical models $[2,3,4]$, in biomembrane modeling $[5,6,7]$, or, more recently, in image processing, for example for automatic contours detection or image segmentation $[8,9,10]$, as well as surface reconstruction and restoration $[11,12,13,14,15]$. In this work, we focus on the numerical approximation of geometric PDEs defined on 3D surfaces, and specifically on two common problems. The first one is the mean curvature flow, for which the considered surface moves in the direction of its mean curvature vector. This causes the surface to evolve towards the minimization of its area [16], and it is of fundamental interest for the study of minimal surfaces. Problems of this kind arise, for example, when studying grain boundary motion in alloys, or modeling physical systems involving surface tension, such as biological cells and membranes, bubbles, capillarity, and others. This problem has been extensively studied theoretically $[16,17,18,19]$ and tackled numerically with different approaches, e.g. by using the Finite Element Method (FEM) in [20, 21], level set formulations in [22, 23], or phase field approaches in [1]. Then, we focus on the Willmore flow problem [24], which leads to the minimization of the Willmore (or bending) energy, which appears, for example, in optimal surface modeling [25], surface restoration [15], and in physical models for biomembranes [26, 27, 28]. Theoretical results about the existence, uniqueness, and regularity of the solutions of the Willmore flow problem can be found in $[29,30,31,32]$. Regarding its numerical approximation ([1]), the seminal work in [33] considers a general surface evolver, which has been applied to the Willmore energy using the FEM. Approximations based on finite difference schemes for axisymmetric solutions are proposed in [34]. In general, the numerical approximation of the Willmore flow on parametric surfaces is based on the FEM, as e.g. in [35, 36, 37]; in [38] a formulation based on the level set method is used, while in [39, 40] approximations of the Willmore flow for curves (also called elastic flow of curves) are studied. For a general review on the numerical approximation of geometric PDEs we refer the reader to [1]; instead, for approximating PDEs on evolving surfaces, we refer to the recent review work [41]. Nevertheless, all these approaches generally involve geometric approximations of quantities which may lead to accuracy issues or complex numerical schemes.

In this work, we focus on geometric PDEs defined on 3D parametric surfaces represented by NURBS geometrical mappings [42]. We spatially discretize the PDEs by means of Isogeometric Analysis (IGA) [43, 44]. Developed with the aim of filling the gap between Computer Aided Design (CAD) and FEM, IGA is a discretization method based on the isogeometric concept for 
which the same basis functions are used for both representing the geometry and constructing the approximation subspace of the PDEs solution. IGA facilitates the exact geometric representation of the computational domain, even at the coarsest level of discretization; moreover, the NURBS function spaces, in which the approximate solution lays, can be enriched by means of refinement procedures that preserve the geometrical representation. Furthermore, NURBS-based IGA permits a fine control over the continuity of the basis functions (over smooth surfaces), allowing the use of globally $C^{k}$-continuous basis functions, with $k \leq p-1$, being $p$ the polynomial degree, this even on closed surfaces $[44,45]$. Other than being particularly accurate and efficient $([46,47,48])$, these high order continuous NURBS bases permit the discretization of high order PDEs within the framework of the standard Galerkin method without resorting to mixed formulations $[45,49,50]$. The advantages of NURBS-based IGA for surface PDEs both of second [51] and high order PDEs are highlighted in [45]. Among these, we have the accurate evaluation of geometric quantities as those arising in geometric PDEs, e.g. the normal to the surface and the mean and Gauss curvatures.

In this paper, we propose NURBS-based IGA for the spatial approximation of geometric PDEs, as the mean curvature and Willmore flows. Moreover, we propose the time discretization of the nonlinear problems arising from the IGA semi-discretization by means of Backward Differentiation Formulas (BDF) $[52,53]$. In particular, we treat the nonlinear terms (including the geometric ones) through time extrapolations compatible with the BDF scheme at hand, leading to the solution at each time step of a linear algebraic system. We provide and critically discuss several numerical results for benchmark problems described by geometric PDEs, which show the accuracy and efficiency of the proposed numerical scheme.

This paper is organized as follows. In Section 2 we introduce the geometric representation and notation used throughout the rest of the paper, together with the definitions of the employed differential operators defined on surfaces. In Section 3 a generic formulation of geometric PDEs is introduced, with further focus on the mean curvature and the Willmore flows. Our approach to the spatial and temporal discretizations of the PDEs, based on IGA and BDF schemes, is presented in Section 4. Numerical results for different test problems are provided and discussed in Section 5. Conclusions follow.

\section{Geometric Representation and Differential Operators on Surfaces}

Let us consider a parametric domain $\widehat{\Omega} \subset \mathbb{R}^{2}$ of finite and positive measure with respect to the topology of $\mathbb{R}^{2}$, together with the parametric coordinate, a vector-valued independent variable 
$\boldsymbol{\xi}=\left(\xi_{1}, \xi_{2}\right) \in \mathbb{R}^{2}$. From here on we denote with $\Omega$ a compact, connected, oriented and smooth surface in $\mathbb{R}^{3}$, with or without boundary $\partial \Omega$, defined by the geometrical mapping $\mathbf{X}$ as:

$$
\mathbf{X}: \widehat{\Omega} \rightarrow \Omega \subset \mathbb{R}^{3}, \quad \boldsymbol{\xi} \rightarrow \mathbf{X}(\boldsymbol{\xi})=\mathbf{p}
$$

By assuming it exists, we denote the inverse mapping as:

$$
\mathbf{X}^{-1}: \Omega \rightarrow \widehat{\Omega} \subset \mathbb{R}^{2}, \quad \mathbf{p} \rightarrow \mathbf{X}^{-1}(\mathbf{p})=\boldsymbol{\xi}
$$

In order to define PDEs on the surface $\Omega$, we introduce surface differential operators on the manifold $\Omega$, e.g. the surface gradient, divergence, and Laplace-Beltrami operators; see e.g. [54, 55, 56]. We introduce some fundamental geometrical quantities associated to the mapping (2.1): in particular, the Jacobian $\widehat{F}: \widehat{\Omega} \rightarrow \mathbb{R}^{3 \times 2}$ of the mapping $\mathbf{X}$, defined as $\widehat{F}_{i, \alpha}(\boldsymbol{\xi}):=\frac{\partial X_{i}}{\partial \xi_{\alpha}}(\boldsymbol{\xi})$, for $i=1,2,3$ and $\alpha=1,2$, the first fundamental form or metric tensor $\widehat{G}: \widehat{\Omega} \rightarrow \mathbb{R}^{2 \times 2}$, defined as $\widehat{G}(\boldsymbol{\xi}):=$ $(\widehat{F}(\boldsymbol{\xi}))^{T} \widehat{F}(\boldsymbol{\xi})$, and its determinant $\widehat{g}: \widehat{\Omega} \rightarrow \mathbb{R}$, defined as $\widehat{g}(\boldsymbol{\xi}):=\sqrt{\operatorname{det}(\widehat{G}(\boldsymbol{\xi}))}$. We consider geometrical mappings (2.1) which are invertible a.e. in $\widehat{\Omega}$, i.e. we allow $\widehat{g}(\boldsymbol{\xi})=0$ only in zeromeasure subsets of $\widehat{\Omega}$, thus requiring $\widehat{g}$ to be positive elsewhere. The geometric quantities $\widehat{F}, \widehat{G}$, and $\widehat{g}$ are therefore expressed directly on the manifold $\Omega$ by using the inverse mapping of Eq. (2.2) as:

$$
F(\mathbf{p}):=\left(\widehat{F} \circ \mathbf{X}^{-1}\right)(\mathbf{p}), \quad G(\mathbf{p}):=\left(\widehat{G} \circ \mathbf{X}^{-1}\right)(\mathbf{p}), \quad \text { and } g(\mathbf{p}):=\left(\widehat{g} \circ \mathbf{X}^{-1}\right)(\mathbf{p}) \quad \text { for } \mathbf{p} \in \Omega .
$$

Moreover, we denote with $\mathbf{n}_{\Omega}: \Omega \rightarrow \mathbb{R}^{3}$ the unit vector normal to the surface $\Omega$. By proceeding as in Eq. (2.3), any sufficiently regular function defined on the manifold $\Omega$, say $\phi \in C^{0}(\Omega)$, is expressed in the parametric domain $\widehat{\Omega}$ as:

$$
\phi(\mathbf{p})=\left(\widehat{\phi} \circ \mathbf{X}^{-1}\right)(\mathbf{p}) \quad \text { for } \mathbf{p} \in \Omega
$$

with $\widehat{\phi}(\boldsymbol{\xi}):=\phi(\mathbf{X}(\boldsymbol{\xi}))$, for $\boldsymbol{\xi} \in \widehat{\Omega}$. By denoting with $\widetilde{\Omega} \subset \mathbb{R}^{3}$ a 3D domain containing the surface $\Omega$ (a "tubular" region) and considering a generic function $\phi \in C^{1}(\Omega)$, we define the surface gradient of $\phi$ as the orthogonal projection of the gradient of its smooth prolongation $\tilde{\phi}$ on $\widetilde{\Omega}$ onto the tangent hyperplane of $\Omega$ :

$$
\nabla_{\Omega} \phi:=\left.\left(\mathrm{I}-\mathbf{n}_{\Omega} \otimes \mathbf{n}_{\Omega}\right) \nabla \tilde{\phi}\right|_{\Omega}
$$

with I being the second order identity tensor. In the same way, we define the surface divergence as $\nabla_{\Omega} \cdot \varphi:=\operatorname{tr}\left(\nabla_{\Omega} \varphi\right)$, with $\varphi \in\left[C^{1}(\Omega)\right]^{3}$, and the Laplace-Beltrami operator as $\Delta_{\Omega} \phi:=\nabla_{\Omega} \cdot \nabla_{\Omega} \phi$ 
provided that $\phi \in C^{2}(\Omega)$. Then, we exploit the geometric quantities defined in Eq. (2.3) to rewrite the gradient and Laplace-Beltrami differential operators in the parametric domain $\widehat{\Omega}$ as $([54,56])$ :

$$
\nabla_{\Omega} \phi(\mathbf{p})=\left(\left[\widehat{F} \widehat{G}^{-1} \widehat{\nabla} \widehat{\phi}\right] \circ \mathbf{X}^{-1}\right)(\mathbf{p}) \quad \text { for } \mathbf{p} \in \Omega
$$

and

$$
\Delta_{\Omega} \phi(\mathbf{p})=\left(\left[\frac{1}{\widehat{g}} \widehat{\nabla} \cdot\left(\widehat{g} \widehat{G}^{-1} \widehat{\nabla} \widehat{\phi}\right)\right] \circ \mathbf{X}^{-1}\right)(\mathbf{p}) \quad \text { for } \mathbf{p} \in \Omega
$$

respectively.

The second fundamental form associated to the geometric mapping (2.1) is the second order tensor defined as:

$$
\mathcal{H}(\mathbf{p}):=\nabla_{\Omega} \mathbf{n}_{\Omega}(\mathbf{p}) \quad \text { for } \mathbf{p} \in \Omega
$$

The symmetric tensor $\mathcal{H}$ is also called shape operator [56]; it possesses a null eigenvalue associated to the eigenvector $\mathbf{n}_{\Omega}$ and two other non zero eigenvalues (since we consider surfaces in $\mathbb{R}^{3}$ ) called principal curvatures and denoted in this work as $\kappa_{i}$, with $i=1,2$. We define the total mean curvature $H$ as the trace of $\mathcal{H}$ :

$$
H(\mathbf{p}):=\operatorname{tr}(\mathcal{H}(\mathbf{p}))=\kappa_{1}(\mathbf{p})+\kappa_{2}(\mathbf{p}) \quad \text { for } \mathbf{p} \in \Omega
$$

We consider the normal $\mathbf{n}_{\Omega}$ to be oriented such that $H$ is positive for spherical surfaces with the normal pointing away from their origin. We define also the total mean curvature vector as

$$
\mathbf{H}(\mathbf{p}):=H(\mathbf{p}) \mathbf{n}_{\Omega}(\mathbf{p}) \quad \text { for } \mathbf{p} \in \Omega
$$

and we denote with $K$ the Gauss curvature, defined as the product of the principal curvatures:

$$
K(\mathbf{p}):=\kappa_{1}(\mathbf{p}) \kappa_{2}(\mathbf{p}) \quad \text { for } \mathbf{p} \in \Omega
$$

Furthermore, we introduce the identity function $\mathbf{x}: \Omega \rightarrow \mathbb{R}^{3}$ on $\Omega$, i.e. the map

$$
\mathbf{x}(\mathbf{X}(\boldsymbol{\xi}))=\mathbf{X}(\boldsymbol{\xi}) \quad \text { for } \boldsymbol{\xi} \in \widehat{\Omega}
$$

We also recall the important relation $([56])$ :

$$
\left(-\Delta_{\Omega} \mathbf{x}\right)(\mathbf{p})=\mathbf{H}(\mathbf{p}) \quad \text { for } \mathbf{p} \in \Omega
$$

which links the surface Laplace-Beltrami operator applied to the identity function on $\Omega$ to the total mean curvature vector. 


\section{Geometric PDEs}

In this section, we firstly introduce the general formulation of geometric PDEs defined on surfaces, then we focus on the mean curvature and Willmore flow problems.

\subsection{General formulation}

A geometric PDE on a surface is an equation describing the evolution of the surface itself. Starting from an initial surface $\Omega_{0}$, identified by the geometrical mapping $\mathbf{X}_{0}: \widehat{\Omega} \rightarrow \mathbb{R}^{3}$, we focus on finding, for all the times $t \in(0, T)$, a family of surfaces identified by their geometrical mapping $\mathbf{X}(t): \widehat{\Omega} \rightarrow \mathbb{R}^{3}$, whose evolution obeys a differential law of the form $\dot{\mathbf{X}}=\mathcal{F}\left(t, \mathbf{X}, \mathbf{n}_{\Omega}, H, K, \ldots\right)$ on $\Omega_{t}$, with $\mathbf{X}(0)=\mathbf{X}_{0}$, and possibly with boundary conditions on $\partial \Omega_{t}$ in case the latter is not empty (that is when $\Omega_{t}$ is not a closed surface). From now on, we indicate with $\Omega_{t} \subset \mathbb{R}^{3}$ the surface identified by the geometrical mapping $\mathbf{X}(t)$ at each time $t \in[0, T]$, and, if it exists, its boundary as $\partial \Omega_{t}$; the subscript $t$ refers to the time dependence of the computational domain, i.e. the evolving surface.

The law $\mathcal{F}$ identifies the problem at hand and potentially depends on several geometrical quantities associated with the geometry. We consider geometric PDEs deriving from the minimization of an energy functional $J(\Omega)$ under certain geometrical constraints. This functional can be seen as the objective functional in an optimization process, where the design variable is represented by the surface $\Omega$ itself. $J$ usually depends on geometrical quantities associated to the geometrical mapping of the surface $\Omega$, as described in Section 2. In this work, we focus on $L^{2}$-gradient flows $[57,58]$ of energy functionals, even if other options are available. We consider the evolving surface $\Omega_{t}$ to be sufficiently smooth both in space and time and described by a smooth geometrical mapping $\mathbf{X}=\mathbf{X}(\boldsymbol{\xi}, t), \boldsymbol{\xi} \in \widehat{\Omega}$, for each $t \in(0, T)$, which actually depends on the form of $J$. Then, following Eq. (2.12) we introduce the identity function:

$$
\mathbf{x}: \Omega_{t} \rightarrow \mathbb{R}^{3} \quad: \quad \mathbf{x}(\mathbf{X}(\boldsymbol{\xi}, t)) \equiv \mathbf{X}(\boldsymbol{\xi}, t), \quad \boldsymbol{\xi} \in \widehat{\Omega}, \quad \text { for all } t \in(0, T)
$$

In order to simplify the notation, henceforth we drop the arguments $\mathbf{X}(\boldsymbol{\xi}, t)$ and $t$, for which we always assume that $\boldsymbol{\xi} \in \widehat{\Omega}$ and $t \in(0, T)$, for quantities defined on $\Omega_{t}$ for all $t \in(0, T)$, as e.g. $\mathbf{x}$. We remark that $\Omega_{t}$ is a function of the mapping $\mathbf{x}(t)$, as $\Omega_{t}=\Omega(\mathbf{x}(t))$. We highlight the fact that, in this work, we only consider geometric PDEs defined on parametrized surfaces: indeed, we do not aim at treating changes of topology, for which we keep the parametric domain $\widehat{\Omega}$ invariant throughout the geometric evolution process. 
We denote with $\mathbf{v}: \Omega_{t} \rightarrow \mathbb{R}^{3}$ and with $v: \Omega_{t} \rightarrow \mathbb{R}$, for all $t \in(0, T)$, the velocity and the normal velocity of the surface $\Omega_{t}$ respectively, reading:

$$
\mathbf{v}:=\frac{\partial \mathbf{X}}{\partial t} \quad \text { and } \quad v:=\mathbf{v} \cdot \mathbf{n}_{\Omega}
$$

respectively. In general, we write the material derivative of the identity function $\mathbf{x}$ as ([36]):

$$
\dot{\mathbf{x}}=\frac{\partial \mathbf{x}}{\partial t}+\mathbf{v} \cdot \nabla \mathbf{x}
$$

which simplifies into:

$$
\dot{\mathbf{x}}=\mathbf{v}=\frac{\partial \mathbf{X}}{\partial t}
$$

since $\frac{\partial \mathbf{x}}{\partial t} \equiv 0$ and $\nabla \mathbf{x}=\mathrm{I}$.

In order to treat the material derivative of quantities depending on volume and area integrals, we use the transport theorem $[1,56]$. Let us consider a function $w \in C^{1}\left(\widetilde{\Omega}_{t}\right)$, where we have introduced an open set $\widetilde{\Omega}_{t}$ such that $\Omega_{t} \subset \widetilde{\Omega}_{t}$ for all $t \in(0, T)$. Under this assumption, we compute the material derivative of the function $w$ integrated in $\Omega_{t}$ as:

$$
\frac{d}{d t} \int_{\Omega_{t}} w d \Omega_{t}=\int_{\Omega_{t}} \frac{\partial w}{\partial t} d \Omega_{t}+\int_{\Omega_{t}} w v H d \Omega_{t}+\int_{\Omega_{t}} \frac{\partial w}{\partial \mathbf{n}_{\Omega}} v d \Omega_{t}
$$

where $H$ is the mean curvature defined in Eq. (2.9). Moreover, by assuming that each surface $\Omega_{t}$ is the boundary of an open bounded subset $\Theta_{t} \subset \mathbb{R}^{3}$, we compute the material derivative of the function $w$ integrated in the volume $\Theta_{t}$ as:

$$
\frac{d}{d t} \int_{\Theta_{t}} w d \Theta_{t}=\int_{\Theta_{t}} \frac{\partial w}{\partial t} d \Theta_{t}+\int_{\Omega_{t}} w v d \Omega_{t} .
$$

We denote with $d J\left(\Omega_{t}\right)(\boldsymbol{\varphi})$ the shape differential of $J$ at fixed time $t \in(0, T)$, i.e. the first variation of $J$ corresponding to a deformation of $\Omega_{t}$ along the direction $\varphi: \Omega_{t} \rightarrow \mathbb{R}^{3}$ ([56]), as:

$$
d J\left(\Omega_{t}\right)(\boldsymbol{\varphi})=d J(\mathbf{x})(\boldsymbol{\varphi})=\left.\frac{d}{d \varepsilon} J(\mathbf{x}+\varepsilon \boldsymbol{\varphi})\right|_{\varepsilon=0}=\left\langle J^{\prime}(\mathbf{x}), \boldsymbol{\varphi}\right\rangle .
$$

By assuming $t \rightarrow+\infty$, the stationary points $\left\{\breve{\Omega}_{\infty}\right\}$ of the energy $J$ are then identified by:

$$
d J\left(\breve{\Omega}_{\infty}\right)(\varphi)=0, \quad \forall \varphi: \breve{\Omega}_{\infty} \rightarrow \mathbb{R}^{3} .
$$

In order to find such minimizers of the energy, we consider the $L^{2}$-gradient flow of the functional $J$. Then, the problem becomes: given an initial surface $\Omega_{0} \subset \mathbb{R}^{3}$, find, for all $t \in(0, T), \Omega_{t}$ such that:

$$
\begin{cases}\dot{\mathbf{x}}=-\mu J^{\prime}(\mathbf{x}) & \text { in } \Omega_{t}, \\ \text { b.c.s } & \text { on } \partial \Omega_{t}, \\ \mathbf{x}(0)=\mathbf{x}_{0} & \text { in } \Omega_{0},\end{cases}
$$


where "b.c.s" stands for boundary conditions and $\mu \in \mathbb{R}^{+}$represents the mobility. Thanks to Eq. (3.7), problem (3.9) can be expressed in weak formulation, for all $t \in(0, T)$, as:

$$
\begin{cases}(\dot{\mathbf{x}}, \varphi)_{L^{2}\left(\Omega_{t}\right)}=-\mu d J(\mathbf{x})(\varphi) & \forall \varphi: \Omega_{t} \rightarrow \mathbb{R}^{3} \text { satisfying the essential b.c.s, } \\ \mathbf{x}(0)=\mathrm{x}_{0} & \text { in } \Omega_{0},\end{cases}
$$

with the natural boundary conditions taken into account in the term $d J(\mathbf{x})(\boldsymbol{\varphi})$. Problem (3.10) is, in general, a highly nonlinear system of PDEs whose steady states correspond to local minima of the energy $J$.

\subsection{Mean curvature flow}

Let us consider the energy functional $J=J_{A}$ defined as:

$$
J_{A}(\Omega):=\int_{\Omega} 1 d \Omega
$$

which corresponds to the area of the surface $\Omega$. Under suitable hypotheses (e.g. $\mathbf{X} \in C^{2}(\widehat{\Omega})$ ), the first variation of $J_{A}$ at $\Omega$ along the direction $\varphi: \Omega \rightarrow \mathbb{R}^{3}$ reads $([16,36])$ :

$$
d J_{A}(\Omega)(\boldsymbol{\varphi})=\int_{\Omega} \mathbf{H} \cdot \boldsymbol{\varphi} d \Omega \quad \forall \boldsymbol{\varphi}: \Omega \rightarrow \mathbb{R}^{3}
$$

where $\mathbf{H}$ is the total mean curvature vector defined in Eq. (2.10). The mean curvature flow is the problem associated with the minimization of the area functional $J_{A}$ by means of a $L^{2}$-gradient flow. Following the prototype Eq. (3.9), the problem reads: given an initial surface $\Omega_{0} \subset \mathbb{R}^{3}$ parametrized by the mapping $\mathbf{X}_{0}: \widehat{\Omega} \rightarrow \mathbb{R}^{3}$, find $\Omega_{t}$ parametrized by $\mathbf{x}: \Omega_{t} \rightarrow \mathbb{R}^{3}$, for all $t \in(0, T)$, such that:

$$
\begin{cases}\dot{\mathbf{x}}=-\mu \mathbf{H}(\mathbf{x}) & \text { in } \Omega_{t}, \\ \text { b.c.s } & \text { on } \partial \Omega_{t}, \\ \mathbf{x}(0)=\mathbf{x}_{0} & \text { in } \Omega_{0}\end{cases}
$$

where $\Omega_{t}=\Omega(\mathbf{x}(t))$, as usual. Problem (3.13) models the evolution of the surface towards the local minimization of its area, as can be verified by using the transport theorem of Eq. (3.5) with $w=1$ and the normal velocity $v=-H$, thus obtaining:

$$
\frac{d}{d t}\left|\Omega_{t}\right|=-\frac{1}{\mu} \int_{\Omega_{t}} v^{2} d \Omega_{t}
$$

where $\left|\Omega_{t}\right|$ is the area of $\Omega_{t}$.

The mean curvature flow problem has been extensively studied both from the theoretical and numerical points of view. Specifically, in [18] existence of a solution is studied for the parametric 
evolution of an initial smooth convex surface without boundary. In [19], the evolution of nonparametric surfaces with boundary is analyzed. The problem has been tackled numerically initially with FEM using linear Lagrange polynomials in [20]; then, more advanced schemes, taking also into account the tangential motion of the mesh, have been considered, as e.g. in [21]. For a general overview of the approximation of the mean curvature flow of curves, surfaces, graphs, level sets, and with phase-field approach, we refer the interested reader to [1].

\subsection{Willmore flow}

Let us consider the Willmore energy functional $J=J_{W}$, defined as:

$$
J_{W}(\Omega):=\frac{1}{2} \int_{\Omega} H^{2} d \Omega
$$

which expresses the bending energy associated to a surface; for more details and properties about the Willmore energy, we refer to [24]. The Willmore flow is the $L^{2}$-gradient flow of $J_{W}$. Under suitable hypotheses, the first variation of $J_{W}$ at $\Omega$ along $\varphi: \Omega \rightarrow \mathbb{R}^{3}$ is given by ([24]):

$$
d J_{W}(\Omega)(\boldsymbol{\varphi})=-\int_{\Omega}\left(\Delta_{\Omega} H-\frac{1}{2} H^{3}+H|\mathcal{H}|^{2}\right) \mathbf{n}_{\Omega} \cdot \boldsymbol{\varphi} d \Omega \quad \forall \boldsymbol{\varphi}: \Omega \rightarrow \mathbb{R}^{3} .
$$

We now consider closed surfaces for this specific problem, for which $\partial \Omega=\emptyset$. Following the prototype Eq. (3.9), the Willmore flow problem reads: given an initial surface $\Omega_{0} \subset \mathbb{R}^{3}$ parametrized by the mapping $\mathbf{X}_{0}: \widehat{\Omega} \rightarrow \mathbb{R}^{3}$, find $\Omega_{t}$ parametrized by $\mathbf{x}: \Omega_{t} \rightarrow \mathbb{R}^{3}$, for all $t \in(0, T)$, such that:

$$
\begin{cases}\dot{\mathbf{x}}=\mu\left(\Delta_{\Omega_{t}} H(\mathbf{x})-\frac{1}{2} H^{3}(\mathbf{x})+H(\mathbf{x})|\mathcal{H}(\mathbf{x})|^{2}\right) \mathbf{n}_{\Omega_{t}} & \text { in } \Omega_{t}, \\ \mathbf{x}(0)=\mathbf{x}_{0} & \text { in } \Omega_{0},\end{cases}
$$

with $\Omega_{t}=\Omega(\mathbf{x}(t))$. In the specific case of $3 \mathrm{D}$ closed surfaces, we can conveniently use the relation $|\mathcal{H}|^{2}=\kappa_{1}^{2}+\kappa_{2}^{2}=H^{2}-2 K$, for which we rewrite problem $(3.17)$, for all $t \in(0, T)$, as:

$$
\begin{cases}\dot{\mathbf{x}}=\mu\left(\Delta_{\Omega_{t}} H(\mathbf{x})+\frac{1}{2} H^{3}(\mathbf{x})-2 H(\mathbf{x}) K(\mathbf{x})\right) \mathbf{n}_{\Omega_{t}} & \text { in } \Omega_{t}, \\ \mathbf{x}(0)=\mathbf{x}_{0} & \text { in } \Omega_{0} .\end{cases}
$$

When the parametric domain is mono-dimensional, $\Omega_{t}$ represents a curve and problem (3.17) corresponds to the elastic flow of curves; global existence in time of a solution for curves in $\mathbb{R}^{n}$ was studied in [59] and [39], for $n=2$ and $n \geq 3$ respectively. For surfaces, which represent the focus of this paper, the Willmore flow problem has been studied analytically mainly on closed surfaces. Existence of a solution up to the finite time $T<+\infty$ for two-dimensional surfaces in $\mathbb{R}^{n}$, with 
$n \geq 3$, is proven in [29], with $T$ depending on the curvature of the initial surface $\Omega_{0}$. Existence and uniqueness of the local solution of problem (3.18) under the hypothesis that the initial geometry $\Omega_{0}$ is a compact, closed, immersed, and orientable $C^{2, \alpha}$-surface in $\mathbb{R}^{3}$ is proven in [32], together with the global existence of the solution in time where $\Omega_{0}$ is "sufficiently" close to a sphere. In [30]

global existence of solutions is shown under the assumption that $\int_{\Omega_{0}}|\stackrel{\mathcal{H}}{ }|^{2}$ is sufficiently small, being $\mathcal{H}$ the trace-free part of the second fundamental form $\mathcal{H}$. In [31] the authors proved that if $\Omega_{0}$ is a smooth immersion of a sphere in $\mathbb{R}^{3}$ and it is such that its Willmore energy $J_{W}\left(\Omega_{0}\right) \leq 16 \pi$, then its Willmore flow smoothly exists for all times and converges to a sphere. This result is numerically verified in [34], where results show that the flow develops singularities in finite time when $\Omega_{0}$ is associated to a Willmore energy $J_{W}\left(\Omega_{0}\right)>16 \pi$.

\section{Space and Time Discretizations}

In this section, we describe our approach to the numerical approximation of the mean curvature and Willmore flow problems of Section 3. Let us consider the general gradient flow problem (3.9) derived from the minimization of an energy functional $J(\Omega)$. Then, we can write the problem in weak form as follows:

$$
\text { find, for all } t \in(0, T), \mathbf{x} \in V_{g, t}:\left\{\begin{array}{l}
m(\dot{\mathbf{x}}, \boldsymbol{\varphi})+a(\mathbf{x})(\boldsymbol{\varphi})=0 \\
\mathbf{x}(0)=\mathbf{x}_{0},
\end{array} \forall \boldsymbol{\varphi} \in V_{0, t}\right.
$$

where $a(\cdot)(\cdot)$ is a form which defines the problem under consideration and $m(\cdot, \cdot)$ is the mass form

$m(\dot{\mathbf{x}}, \boldsymbol{\varphi})=\int_{\Omega_{t}} \dot{\mathbf{x}} \cdot \boldsymbol{\varphi} d \Omega_{t}$. The Hilbert spaces $V_{g, t}$ and $V_{0, t}$ depend on the order of the spatial differential operators involved in the form $a(\cdot)(\cdot)$; in case the surface $\Omega_{t}$ is open, $V_{g, t}$ accounts for the non-homogeneous essential boundary conditions and $V_{0, t}$ is its homogeneous counterpart. If the problem is defined on closed surfaces, then $\partial \Omega_{t} \equiv \emptyset$ for all $t \in(0, T)$ and the spaces $V_{0, t}$ and $V_{g, t}$ coincide and are identified with the Hilbert space $V_{t}$. We remark that the forms $a(\cdot)(\cdot)$ and $m(\cdot, \cdot)$ and the function spaces depend on the current computational domain $\Omega_{t}$, which itself depends on $\mathbf{x}(t)$, as well as the geometric quantities in the form $a(\cdot)(\cdot)$. In Sections 4.1 and 4.2 we introduce the spatial and time discretization techniques adopted to approximate problem (4.1), respectively.

\subsection{Isogeometric Analysis}

We spatially discretize problem (4.1) by means of NURBS-based Isogeometric Analysis (IGA) in the framework of the Galerkin method ([44]). 


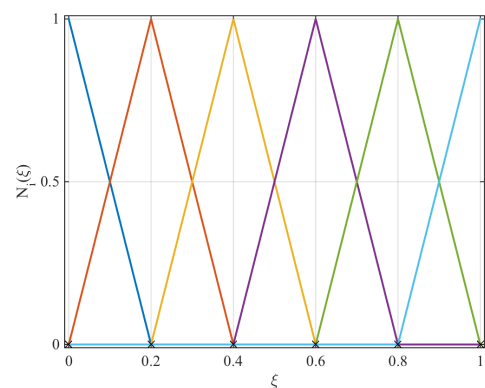

(a) $p=1$

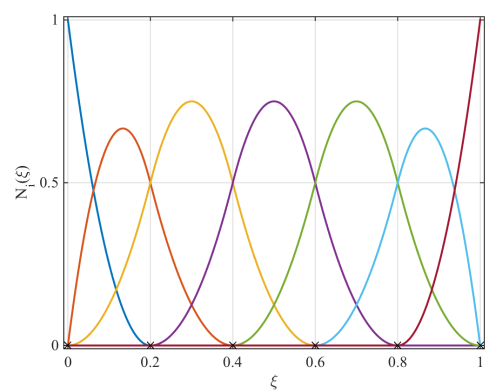

(b) $p=2$

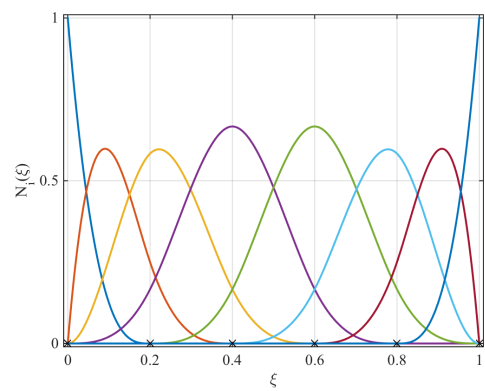

(c) $p=3$

Figure 1: Univariate B-spline basis functions of polynomial degrees $p=1,2$, and 3 obtained from the knot vectors $\Xi=\left\{\{0\}^{p+1}, \frac{1}{5}, \frac{2}{5}, \frac{3}{5}, \frac{4}{5},\{1\}^{p+1}\right\} ;$ the basis functions are globally $C^{p-1}$-continuous in $\widehat{\Omega}=(0,1)$.

\subsubsection{NURBS}

In this work, we specifically consider surfaces represented by NURBS, as in [45]; for further details and properties of NURBS, see [42,43]. The geometrical mapping (2.1) in the case of NURBS surfaces reads:

$$
\mathbf{X}(\boldsymbol{\xi})=\sum_{i=1}^{n_{b f}} \widehat{R}_{i}(\boldsymbol{\xi}) \mathbf{P}_{i}, \quad \widehat{R}_{i}(\boldsymbol{\xi}):=\frac{w_{i}}{\sum_{j=1}^{n_{b f}} w_{j} \widehat{N}_{j}(\boldsymbol{\xi})} \widehat{N}_{i}(\boldsymbol{\xi}) \quad \text { for } i=1, \ldots, n_{b f},
$$

with $\mathbf{P}_{i} \in \mathbb{R}^{3}$, for $i=1, \ldots, n_{b f}$, being the control points in the physical space where the surface $\Omega$ is defined and $n_{b f}$ the number of basis functions. The NURBS basis functions $\widehat{R}_{i}(\boldsymbol{\xi})$, for $i=1, \ldots, n_{b f}$, are obtained from the B-spline basis functions $\widehat{N}_{i}(\boldsymbol{\xi})$ by projective transformations, with weights $w_{i} \in \mathbb{R}$, for $i=1, \ldots, n_{b f}$. This allows the NURBS mapping to exactly represent surfaces such as conic sections, e.g. spheres and tori, which are geometrical entities often considered with geometric PDEs. Univariate B-spline basis functions are built using the Cox-de Boor recursion formula [42] with the knot vectors $\Xi:=\left\{\xi_{j}\right\}_{j=1}^{n_{b f}+p+1}$, where $p$ is the polynomial degree, $\xi_{j} \in \mathbb{R}$ are the knots, and the intervals between the knots are called knot spans. The multiplicity of a knot inside the knot vector controls the continuity of the basis functions across that knot. In practice, repeating a knot $k$ times makes the basis functions to be $C^{p-k}$-continuous across that knot. In Figure 1, we report examples of B-spline basis functions with different polynomial degrees and order of continuities.

By considering a bidimensional parametric domain and denoting with $\alpha=1,2$ the parametric directions, the multivariate B-spline basis functions $\widehat{N}_{i}(\boldsymbol{\xi})$ are built from the tensor product of the univariate B-spline basis functions $\widehat{N}_{i, \alpha}$, for $i=1, \ldots, n_{b f, \alpha}$, built using the knot vectors $\Xi_{\alpha}$, for $\alpha=1,2$. The tensor product of knot vectors also defines a partition of the parametric domain $\widehat{\Omega}$ 
into regions defined by the knot spans, also called mesh elements.

\subsubsection{NURBS-based IGA}

IGA is an approximation method for PDEs based on the isoparametric concept, for which the same basis functions used for the geometric representation are then also used for the numerical approximation of the PDEs [43, 44].

The NURBS computational domain, i.e. the surface $\Omega$, at a prescribed time instance is represented at the coarsest level of discretization. From Eq. (4.2) we define the NURBS function space $\widehat{\mathcal{N}}_{h}$ over the parametric domain $\widehat{\Omega}$, and the function space $\mathcal{N}_{h}$ over the physical domain $\Omega$, as:

$$
\widehat{\mathcal{N}}_{h}:=\operatorname{span}\left\{\widehat{R}_{i}, \quad i=1, \ldots, n_{b f}\right\} \quad \text { and } \quad \mathcal{N}_{h}:=\operatorname{span}\left\{\widehat{R}_{i}(\boldsymbol{\xi}) \circ \mathbf{X}^{-1}(\boldsymbol{\xi}), \quad i=1, \ldots, n_{b f}\right\},
$$

respectively. These spaces are used to build the trial function spaces for the approximation of PDEs and, since we consider a Galerkin method, also the test function spaces. We denote with the subscript $h$ the mesh elements' characteristic size, being usually defined as the maximum diameter of the elements in the physical space [51]. The function spaces of Eq. (4.3) can be enriched with several refinement procedures [43], which maintain the geometrical representation unaffected. In particular, with the knot insertion new knots are introduced into the knot vectors, creating possibly new knot spans and increasing the total number of mesh elements and basis functions. If during the knot insertion the continuity of the basis functions is preserved, this procedure is analogous to the h-refinement of the FEM. The order elevation procedure increases the polynomial degree

$p$ of the NURBS basis functions while preserving the existing continuity of the basis functions, in a way analogous to the p-refinement of the FEM. Finally, k-refinement consists in the sequential application of order elevation and knot insertion procedures in order to increase the polynomial degree $p$ while maintaining the highest possible degree of continuity of the basis functions across the mesh elements, a procedure which does not have an analogous counterpart in the FEM. For more details, see e.g. [42, 43, 44].

Let us consider the general gradient flow problem in weak form of Eq. (4.1). Since we deal with surfaces defined by geometrical mappings in the form (2.1), which are invertible a.e., we pull-back problem (4.1) into the parametric domain $\widehat{\Omega}$, thus obtaining:

$$
\text { find, for all } t \in(0, T), \mathbf{X} \in \widehat{V}_{g} \quad:\left\{\begin{array}{l}
\widehat{m}(\dot{\mathbf{X}}, \widehat{\boldsymbol{\varphi}})+\widehat{a}(\mathbf{X})(\widehat{\boldsymbol{\varphi}})=0 \\
\mathbf{X}(0)=\mathbf{X}_{0}
\end{array} \quad \forall \widehat{\boldsymbol{\varphi}} \in \widehat{V}_{0},\right.
$$


where $\widehat{V}_{g}$ and $\widehat{V}_{0}$ correspond to the "pull-back" of the function spaces $V_{g, t}$ and $V_{0, t}$ on the parametric domain $\widehat{\Omega}$, respectively; the forms $\widehat{m}(\cdot, \cdot)$ and $\widehat{a}(\cdot)(\cdot)$ result from the pull-back operation applied to $m(\cdot, \cdot)$ and $a(\cdot)(\cdot)$, respectively. In particular, we have:

$$
\widehat{m}(\dot{\mathbf{X}}, \widehat{\varphi})=\int_{\widehat{\Omega}} \dot{\mathbf{X}} \cdot \widehat{\varphi} \widehat{g} d \widehat{\Omega}
$$

for $\widehat{\varphi} \in \widehat{V}_{0}$, where $\widehat{g}$ is the determinant of the first fundamental form of the mapping defined in Section 2. The form $a(\cdot)(\cdot)$ is pulled-back in a similar fashion, using the geometric quantities introduced in Section 2 and will be specified for the gradient flow problem under consideration.

Then, we proceed with the spatial discretization of problem (4.4). We choose suitable NURBS function spaces for the trial and test functions, accordingly with the NURBS mapping, for which, for all $t \in(0, T)$, we seek solutions in the form:

$$
\mathbf{x}_{h}(t)=\sum_{i=1}^{n_{b f}}\left(\widehat{R}_{i} \circ \mathbf{X}^{-1}\right) \mathbf{P}_{i}(t)
$$

in the physical space, where $\mathbf{P}_{i}(t)$, for $i=1, \ldots, n_{b f}$, are the control points introduced in Section 2 which describe the geometry and, in this context of geometric PDEs, also play the role of vectorvalued control variables. The semi-discretized problem reads:

$$
\text { find, for all } t \in(0, T), \mathbf{x}_{h} \in V_{g, t, h}:\left\{\begin{array}{l}
m\left(\dot{\mathbf{x}}_{h}, \boldsymbol{\varphi}_{h}\right)+a\left(\mathbf{x}_{h}\right)\left(\boldsymbol{\varphi}_{h}\right)=0 \quad \forall \boldsymbol{\varphi}_{h} \in V_{0, t, h}, \\
\mathbf{x}_{h}(0)=\mathbf{x}_{0, h},
\end{array}\right.
$$

which can be pulled-back into the parametric domain $\widehat{\Omega}$, thus obtaining:

find, for all $t \in(0, T), \mathbf{X}_{h} \in \widehat{V}_{g, h}:\left\{\begin{array}{l}\widehat{m}\left(\dot{\mathbf{X}}_{h}, \widehat{\boldsymbol{\varphi}}_{h}\right)+\widehat{a}\left(\mathbf{X}_{h}\right)\left(\widehat{\boldsymbol{\varphi}}_{h}\right)=0 \quad \forall \widehat{\boldsymbol{\varphi}}_{h} \in \widehat{V}_{0, h}, \\ \mathbf{X}_{h}(0)=\mathbf{X}_{0, h} ;\end{array}\right.$

the finite-dimensional function spaces $V_{g, t, h}, V_{0, t, h}, \widehat{V}_{g, h}$ and $\widehat{V}_{0, h}$ are subsets of NURBS function spaces defined as $V_{g, t, h}:=V_{g, t} \cap\left[\mathcal{N}_{h}\right]^{3}, V_{0, t, h}:=V_{0, t} \cap\left[\mathcal{N}_{h}\right]^{3}, \widehat{V}_{g, h}:=\widehat{V}_{g} \cap\left[\widehat{\mathcal{N}}_{h}\right]^{3}$ and $\widehat{V}_{0, h}:=\widehat{V}_{0} \cap\left[\widehat{\mathcal{N}}_{h}\right]^{3}$, respectively.

While simple surfaces can straightforwardly be represented by using $C^{p-1}$-continuous NURBS basis functions in a single patch, this may be not the case of more complex surfaces, as the closed ones, for which the single patch NURBS representation involves bases which are only globally $C^{0}$-continuous in $\Omega$ and $\widehat{\Omega}$. Since we are interested in the approximation of geometric PDEs of order equal or eventually higher than two (as the Willmore flow), we need to use trial and test function spaces with the Galerkin method which guarantee high order (at least 1) global continuity 
of the basis functions over the whole surface. Therefore, when dealing with closed surfaces, we consider the construction of periodic NURBS function spaces $\mathcal{N}_{h}^{\text {per }}$, built from the original NURBS function space $\mathcal{N}_{h}$, defining $\Omega$, through element-wise linear transformations of the basis functions and suitable constraints among the degrees of freedom $[49,45]$. In particular, we apply the linear operator $\mathbf{T}^{\text {per }} \in \mathbb{R}^{n_{b f} \times n_{b f}}$ to the basis functions $\widehat{\mathbf{R}}:=\left\{\widehat{R}_{i}\right\}_{i=1}^{n_{b f}}$ defining the NURBS function space $\widehat{\mathcal{N}}_{h}$, thus obtaining $\widehat{\mathbf{R}}^{\text {per }}:=\mathbf{T}^{\text {per }} \widehat{\mathbf{R}}(\boldsymbol{\xi})$ and the periodic spaces $\widehat{\mathcal{N}}_{h}^{\text {per }}$ and $\mathcal{N}_{h}^{\text {per }}$ :

$$
\begin{aligned}
\widehat{\mathcal{N}}_{h}^{\text {per }} & :=\operatorname{span}\left\{\widehat{R}_{i}^{\text {per }}, \quad i=1, \ldots, n_{b f}\right\}, \\
\mathcal{N}_{h}^{\text {per }} & :=\operatorname{span}\left\{\widehat{R}_{i}^{\text {per }}(\boldsymbol{\xi}) \circ \mathbf{x}^{-1}(\boldsymbol{\xi}), \quad i=1, \ldots, n_{b f}\right\} .
\end{aligned}
$$

For more details on the periodic NURBS function spaces and error analysis about the IGA approximation of high order PDEs defined on closed surfaces, we refer the interested reader to [45]. We remark that, since the control points $\left\{\mathbf{P}_{i}\right\}_{i=1}^{n_{b f}}$ describe the geometry but at the same time represent the unknown of the problem, we cannot use a subparametric approach as in [45]. Indeed, we also need to apply the same transformations to the control points in order to use the same NURBS function space for both the solution and the geometrical representation, i.e. a pure isoparametric approach. Being $\mathbf{P}_{i}^{\text {per }} \in \mathbb{R}^{3}, i=1, \ldots, n_{b f}$ the transformed control points, obtained with the following relation $([49])$ :

$$
\mathbf{P}^{\text {per }}:=\left(\mathbf{T}^{\text {per }}\right)^{-T} \mathbf{P}
$$

we stress the fact that the representation of $\Omega$ given by the periodic NURBS basis functions $\widehat{\mathbf{R}}^{\text {per }}$ together with the control points $\mathbf{P}^{\text {per }}$ is equivalent to the one given by the original NURBS basis functions $\widehat{\mathbf{R}}$ with the control points $\mathbf{P}$, i.e. $\mathbf{x}_{h}=\sum_{i=1}^{n_{b f}} \widehat{R}_{i}^{p e r} \mathbf{P}_{i}^{p e r}=\sum_{i=1}^{n_{b f}} \widehat{R}_{i} \mathbf{P}_{i}$. Therefore, when dealing with closed or partially closed geometries, the quantities $\widehat{\mathbf{R}}^{\text {per }}, \mathbf{P}^{\text {per }}$ and $\mathcal{N}_{h}^{\text {per }}$ are substituted into the original ones in the definition of the problem to be approximated, as in Eqs. (4.6), (4.7), and (4.8). In order to simplify the notations, from now on we will drop the superscript "per", referring indifferently to both the non-transformed or the transformed NURBS function space and control points depending on the situation at hand (open or closed surfaces).

\subsubsection{IGA for mean curvature flow}

Let us consider the mean curvature flow problem of Eq. (3.13). By assuming sufficient regularity for all the geometric quantities involved, by using the relation of Eq. (2.13) and integrating by parts the Laplace-Beltrami operator, we recast the mean curvature problem (3.13) in the general 
formulation of Eq. (4.1) with the form $a(\cdot)(\cdot)$ being defined as $([20])$ :

$$
a_{M C F}(\mathbf{x})(\varphi):=\mu \int_{\Omega} \nabla_{\Omega} \mathbf{x}: \nabla_{\Omega} \varphi d \Omega
$$

for $\varphi \in V_{0}$, with $V_{0}$ being subset of $\left[H^{1}(\Omega)\right]^{3}$. The semi-discretized problem obtained by the NURBS-based IGA approximation of the mean curvature flow problem is in the form of Eq. (4.7), with the function spaces $V_{g, t, h}$ and $V_{0, t, h}$ being subsets of $V_{t, h}:=\left[H^{1}\left(\Omega_{t}\right)\right]^{3} \cap \mathcal{N}_{h}$. When the semidiscretized problem is rewritten into the parametric domain $\widehat{\Omega}$ after the pull-back operation, the form of Eq. (4.8) becomes:

$$
\widehat{a}\left(\mathbf{X}_{h}\right)\left(\widehat{\boldsymbol{\varphi}}_{h}\right):=\mu \int_{\widehat{\Omega}}\left(\widehat{F} \widehat{G}^{-1} \widehat{\nabla} \mathbf{X}_{h}\right):\left(\widehat{F} \widehat{G}^{-1} \widehat{\nabla} \widehat{\varphi}_{h}\right) \widehat{g} d \widehat{\Omega}
$$

for $\widehat{\varphi}_{h} \in \widehat{V}_{0, h}$, having used the relation in Eq. (2.6), with the function spaces $\widehat{V}_{g, h}$ and $\widehat{V}_{0, h}$ being subsets of $\widehat{V}_{h}:=\left[H^{1}(\widehat{\Omega})\right]^{3} \cap\left[\widehat{\mathcal{N}}_{h}\right]^{3}$.

\subsubsection{IGA for Willmore flow}

The Willmore flow problem of Eq. (3.18) is a nonlinear time dependent high order PDE. For spatial discretizations based on the standard FEM with $C^{0}$-continuous basis functions, mixed formulations to decrease the order of the PDE are commonly used $[5,35,36]$. In addition, the term $H K$, which involves both the mean $(H)$ and the Gauss $(K)$ curvatures, nonlinearly depends on the principal curvatures and it is difficult to treat with variational methods [36]; therefore, terms as $K$ or the normal to the surface $\mathbf{n}_{\Omega}$ are usually avoided through suitable manipulations in the weak formulation of the problem. Regardless of the order of the differential problem, these considerations lead to the adoption of mixed formulations where additional unknowns are introduced, usually being the velocity $\mathbf{v}$, the normal component of the velocity $v$ along $\mathbf{n}_{\Omega}$, the mean curvature $H$, or the mean curvature vector $\mathbf{H}[5,35,36,37]$, for which the resulting PDEs are of the second order.

In the framework of NURBS-based IGA, one benefits both from the exact representation of the geometry $\Omega$, with the possibility of calculating the geometrical quantities directly from the NURBS representation, and the ability to treat high order surface PDE in a straightforward manner [45]. We therefore propose the following weak formulation of the Willmore flow problem: 
find, for all $t \in(0, T), \mathbf{x} \in V_{t}$ and $v \in W_{t} \quad:$

$$
\left\{\begin{array}{l}
\int_{\Omega_{t}} v \psi d \Omega_{t}+\mu \int_{\Omega_{t}}\left(\Delta_{\Omega_{t}} \mathbf{x} \cdot \mathbf{n}_{\Omega_{t}}\right) \Delta_{\Omega_{t}} \psi d \Omega_{t} \\
\quad+\mu \int_{\Omega_{t}}\left(\frac{1}{2} H^{2}-2 K\right)\left(\Delta_{\Omega_{t}} \mathbf{x} \cdot \mathbf{n}_{\Omega_{t}}\right) \psi d \Omega_{t}=0 \quad \forall \psi \in W_{t}, \\
\int_{\Omega_{t}} \dot{\mathbf{x}} \cdot \boldsymbol{\varphi} d \Omega_{t}-\int_{\Omega_{t}} v \mathbf{n}_{\Omega_{t}} \cdot \boldsymbol{\varphi} d \Omega_{t}=0 \quad \forall \boldsymbol{\varphi} \in V_{t}, \\
\mathbf{x}(0)=\mathbf{x}_{0}, \\
v(0)=v_{0},
\end{array}\right.
$$

where $V_{t}:=\left[H^{2}\left(\Omega_{t}\right)\right]^{3}$, while $W_{t}:=H^{2}\left(\Omega_{t}\right)$; the normal velocity $v$ (defined in Eq. (3.2)) is also an unknown of the problem. We consider NURBS-based IGA for the approximation of (4.13). We therefore discretize the equations following the same procedure described in Section 4.1.2, seeking the trial and test functions for the unknowns $\mathbf{x}_{h}$ and $v_{h}$ in the function spaces $V_{t, h}:=V_{t} \cap\left[\mathcal{N}_{h}\right]^{3}$ and $W_{t, h}:=W_{t} \cap \mathcal{N}_{h}$, respectively. We remark that, with IGA, the evaluation of the terms involving $H$ and $K$ is straightforward, since the curvatures can be computed directly and "exactly" from the NURBS mapping $\mathbf{x}_{h}$. Problem (4.13) is rewritten into the parametric domain $\widehat{\Omega}$ through a pull-back operation as described in Section 4.1.2 and similarly to the approximation of the mean curvature flow problem of Section 4.1.3; in this case, we also use the relation of Eq. (2.7) for the treatment of the surface Laplace-Beltrami operator in the parametric domain $\widehat{\Omega}$. We remark that, since we need to ensure that the test and trial function spaces are subsets of $H^{2}$, we consider NURBS function spaces with basis functions at least globally $C^{1}$-continuous a.e. in $\Omega_{t}$, for all $t \in(0, T)$. Moreover, since $\Omega$ is a closed surface, we consider NURBS periodic function spaces, as mentioned in Section 4.1.2.

\subsection{Time discretization}

Problems governed by geometric PDEs are generally nonlinear. Indeed, all the geometric quantities and differential operators involved in problem (4.1) defined in $\Omega$ depend themselves on $\mathbf{x}$. In literature $[5,20,36]$, such problems are typically discretized in time with a semi-implicit first order scheme with an explicit treatment of the geometric nonlinear terms; in this manner, at any given time step, all the geometric quantities and differential operators are evaluated using the solution obtained at the previous time steps. In this paper, we propose the time discretization of geometric PDEs with high order implicit Backward Differentiation Formulas (BDF) [52]. We address the 
circular dependence between the solution and the geometric quantities by treating the geometry explicitly, using the solution extrapolated from the previous time steps; see e.g. [60,61].

Let us consider the time discretization of the spatially discretized problem (4.7) in the time interval $[0, T]$, with $n$ as time step index and $t_{n}$ the $n$-th time step, such that $t_{0}=0$ and $t_{N}=T$. We introduce the approximate surface $\Omega_{n+1}$ as the surface defined by the NURBS mapping:

$$
\mathbf{X}_{h}^{n+1}(\boldsymbol{\xi})=\sum_{i=1}^{n_{b f}} \widehat{R}_{i}(\boldsymbol{\xi}) \mathbf{P}_{i}^{n+1}
$$

from Eq. (4.2), where $\left\{\mathbf{P}_{i}^{n+1}\right\}_{i=1}^{n_{b f}}$ are the control points computed at the time instance $t_{n+1}$. In general, when considering a fixed time step size $\Delta t$, the time discretization using a $k$-th order BDF scheme consists in approximating the time derivative $\dot{\mathbf{X}}_{h}$ at time step $n+1$ through a linear combination of the mappings $\mathbf{X}_{h}$ at the time step $n+1$ and the $k$ previous time steps, as:

$$
\dot{\mathbf{X}}_{h}^{n+1} \simeq \frac{1}{\Delta t}\left(\alpha_{0} \mathbf{X}_{h}^{n+1}-\sum_{i=1}^{k} \alpha_{i} \mathbf{X}_{h}^{n+1-i}\right)
$$

for $n \geq k-1$, with the coefficients $\alpha_{i} \in \mathbb{R}$, for $i=0, \ldots, k$, being chosen in a way to guarantee that the approximation is of order $k$. Moreover, $\Omega_{*}$, which we refer as the extrapolated surface, is defined by the NURBS mapping:

$$
\mathbf{X}_{h}^{*}(\boldsymbol{\xi})=\sum_{i=1}^{n_{b f}} \widehat{R}_{i}(\boldsymbol{\xi}) \mathbf{P}_{i}^{*}
$$

where $\left\{\mathbf{P}_{i}^{*}\right\}_{i=1}^{n_{b f}}$ are the control points obtained from the sets $\left\{\mathbf{P}_{i}^{n+1-k}\right\}_{i=1}^{n_{b f}}, \ldots,\left\{\mathbf{P}_{i}^{n}\right\}_{i=1}^{n_{b f}}$ with a $k$-th order extrapolation, as ([60]):

$$
\mathbf{P}_{i}^{*}:=\sum_{j=1}^{k} \beta_{j} \mathbf{P}_{i}^{n+1-j}
$$

for $i=1, \ldots, n_{b f}$, with appropriate coefficients $\beta_{j} \in \mathbb{R}$, for $j=1, \ldots, k$. By referring now to the time derivative $\dot{\mathbf{x}}_{h}$ at time step $n+1$ of the identity function $\mathbf{x}_{h}$, following Eqs. (4.14), (4.15), and (4.16), we approximate it with the $k$-th order BDF scheme as:

$$
\dot{\mathbf{x}}_{h}^{n+1} \simeq \frac{1}{\Delta t}\left[\alpha_{0} \mathbf{x}_{h}^{n+1}-\sum_{i=1}^{k} \alpha_{i}\left(\mathbf{x}_{h}^{n+1-i} \circ \mathbf{X}_{h}^{n+1-i}\right) \circ\left(\mathbf{X}_{h}^{*}\right)^{-1}\right]
$$

for $n \geq k-1$. For notational convenience, we define $\mathbf{x}_{h}^{b d f}: \Omega_{*} \rightarrow \mathbb{R}^{3}$ as:

$$
\mathbf{x}_{h}^{b d f}:=\sum_{i=1}^{k} \frac{\alpha_{i}}{\alpha_{0}}\left(\mathbf{x}_{h}^{n+1-i} \circ \mathbf{X}_{h}^{n+1-i}\right) \circ\left(\mathbf{X}_{h}^{*}\right)^{-1}
$$


and $\mathbf{v}_{h}^{n+1}: \Omega_{*} \rightarrow \mathbb{R}^{3}$ as:

$$
\mathbf{v}_{h}^{n+1}:=\alpha_{0} \frac{\mathbf{x}_{h}^{n+1}-\mathbf{x}_{h}^{b d f}}{\Delta t},
$$

as well as the extrapolated solution $\mathbf{x}_{h}^{*}: \Omega_{*} \rightarrow \mathbb{R}^{3}$ at time $t_{n+1}$ following Eqs. (4.16) and (4.17):

$$
\mathbf{x}_{h}^{*}:=\sum_{j=1}^{k} \beta_{j}\left(\mathbf{x}_{h}^{n+1-j} \circ \mathbf{X}_{h}^{n+1-j}\right) \circ\left(\mathbf{X}_{h}^{*}\right)^{-1},
$$

for $n \geq k-1$.

We can therefore rewrite problem (4.7) with respect to the unknown velocity $\mathbf{v}_{h}^{n+1}$ as follows:

$$
\begin{aligned}
& \text { find, for } n=0, \ldots, N-1, \mathbf{x}_{h}^{n+1} \in V_{g, h}^{*}: \\
& \qquad\left\{\begin{array}{l}
m_{h}^{*}\left(\mathbf{v}_{h}^{n+1}, \boldsymbol{\varphi}_{h}\right)+\frac{\Delta t}{\alpha_{0}} a_{h}^{*}\left(\mathbf{v}_{h}^{n+1}, \varphi_{h}\right)=-a_{h}^{*}\left(\mathbf{x}_{h}^{b d f}, \varphi_{h}\right) \quad \forall \varphi_{h} \in V_{0, h}^{*}, \\
\mathbf{v}_{h}^{0}=\mathbf{v}_{0, h},
\end{array}\right.
\end{aligned}
$$

where $V_{g, h}^{*}$ and $V_{0, h}^{*}$ are function spaces defined in the extrapolated surface $\Omega_{*}$, which correspond to $V_{g, t, h}$ and $V_{0, t, h}$ onto $\Omega_{*}$, respectively, and $m_{h}^{*}(\cdot, \cdot)$ and $a_{h}^{*}(\cdot, \cdot)$ are bilinear forms in which the differential operators and domain of integrations are defined in $\Omega_{*}$. For example, the form $m_{h}^{*}(\cdot, \cdot)$ reads:

$$
m_{h}^{*}\left(\mathbf{v}_{h}^{n+1}, \boldsymbol{\varphi}_{h}\right):=\int_{\Omega_{*}} \mathbf{v}_{h}^{n+1} \cdot \boldsymbol{\varphi}_{h} d \Omega_{*} .
$$

The new mapping $\mathbf{x}_{h}^{n+1}: \Omega_{*} \rightarrow \mathbb{R}^{3}$ is then obtained as:

$$
\mathbf{x}_{h}^{n+1}=\mathbf{x}_{h}^{b d f}+\frac{\Delta t}{\alpha_{0}} \mathbf{v}_{h}^{n+1}
$$

corresponding to the new geometrical mapping $\mathbf{X}_{h}^{n+1}$, which defines the new surface $\Omega_{n+1}$ as in Eq. (4.14) and approximating $\Omega_{t_{n+1}}$.

Regarding the mean curvature flow, the full discrete problem is the same of Eq. (4.22), with the form $a_{h}^{*}(\cdot, \cdot)$ defined as:

$$
a_{h}^{*}\left(\mathbf{z}_{h}^{n}, \varphi_{h}\right)=\mu \int_{\Omega_{*}} \nabla_{\Omega_{*}} \mathbf{z}_{h}^{n}: \nabla_{\Omega_{*}} \varphi_{h} d \Omega_{*}
$$


for $\varphi_{h} \in V_{0, h}^{*}$. Instead, for the Willmore flow problem (4.13), the full discrete problem reads:

find, for $n=0, \ldots, N-1, \mathbf{v}_{h}^{n+1} \in V_{h}^{*}$ and $v_{h}^{n+1} \in W_{h}^{*} \quad:$

$$
\left\{\begin{aligned}
\int_{\Omega_{*}} v_{h}^{n+1} \psi_{h} d \Omega_{*}+\mu \frac{\Delta t}{\alpha_{0}} \int_{\Omega_{*}}\left(\Delta_{\Omega_{*}} \mathbf{v}_{h}^{n+1} \cdot \mathbf{n}_{\Omega_{*}}\right) \Delta_{\Omega_{*}} \psi_{h} d \Omega_{*} & \\
+\mu \frac{\Delta t}{\alpha_{0}} \int_{\Omega_{*}}\left[\frac{1}{2}\left(H^{*}\right)^{2}-2 K^{*}\right]\left(\Delta_{\Omega_{*}} \mathbf{v}_{h}^{n+1} \cdot \mathbf{n}_{\Omega_{*}}\right) \psi_{h} d \Omega_{*} & \\
=-\mu \int_{\Omega_{*}}\left(\Delta_{\Omega_{*}} \mathbf{x}_{h}^{b d f} \cdot \mathbf{n}_{\Omega_{*}}\right) \Delta_{\Omega_{*}} \psi_{h} d \Omega_{*} & \\
\quad-\mu \int_{\Omega_{*}}\left[\frac{1}{2}\left(H^{*}\right)^{2}-2 K^{*}\right]\left(\Delta_{\Omega_{*}} \mathbf{x}_{h}^{b d f} \cdot \mathbf{n}_{\Omega_{*}}\right) \psi_{h} d \Omega_{*} & \forall \psi_{h} \in W_{h}^{*}, \\
\int_{\Omega_{*}} \mathbf{v}_{h}^{n+1} \cdot \varphi_{h} d \Omega_{*}-\int_{\Omega_{*}} v_{h}^{n+1} \mathbf{n}_{\Omega_{*}} \cdot \boldsymbol{\varphi}_{h} d \Omega_{*}=0 & \\
v_{h}^{0}=v_{0, h}, & \\
\mathbf{v}_{h}^{0}=\mathbf{v}_{0, h}, &
\end{aligned}\right.
$$

where $V_{h}^{*}$ and $W_{h}^{*}$ are the function spaces $V_{t, h}$ and $W_{t, h}$ built on $\Omega_{*}$, respectively. We remark that the problems (4.22) and (4.26) are solved by recasting them into the parametric domain $\widehat{\Omega}$.

\section{Numerical Results}

In this section, we present several results on the numerical approximation of the mean curvature and the Willmore flow problems on different geometries.

\subsection{Mean curvature flow}

We consider the numerical approximation of the mean curvature flow problem of Eq. (3.13) using the numerical scheme (4.22) proposed in Section 4.1.3. For all the tests we set $\mu=1$ (see Eq. (3.13)).

Test 5.1.1. We consider the mean curvature flow of an initial unit sphere $\Omega_{0}$. By recalling Eq. (3.13) and by exploiting the radial symmetry of the sphere, the geometry $\Omega_{t}$ remains a sphere for each $t \in(0, T)$ with evolution in spherical coordinates described by the following ordinary differential equation [34]:

$$
\left\{\begin{array}{l}
\dot{r}=-\frac{2}{r} \\
r(0)=r_{0}
\end{array}\right.
$$




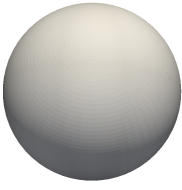

(a) $t=0$

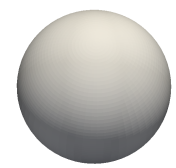

(b) $t=0.062$

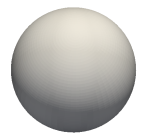

(c) $t=0.125$

(d) $t=0.187$

(e) $t=0.249$

Figure 2: Test 5.1.1. Mean curvature flow of a sphere. Solution at different time instances.

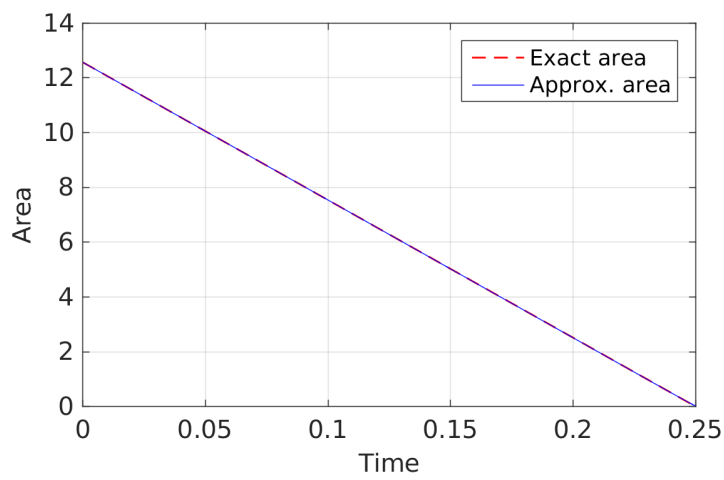

Figure 3: Test 5.1.1. Mean curvature flow of a sphere. Evolution of the approximated area $\left|\Omega_{n}\right|$ and exact area $\left|\Omega_{t_{n}}\right|$ vs. time $t$; NURBS of degree $p=2$ and $C^{1}$-continuous a.e. with 220 mesh elements are used.

where $r(t)$ is the radius of the sphere at time $t$ and $r_{0}$ the radius of $\Omega_{0}$. This equation has analytical solution:

$$
r(t)=\sqrt{r_{0}^{2}-4 t} \quad \text { for } t \in[0, T]
$$

from which it is evident that the sphere degenerates for $t=\frac{r_{0}^{2}}{4}$. Thus, considering an initial sphere $\Omega_{0}$ of radius $r_{0}=1$, we expect the solution of problem (3.13) to be represented by a shrinking sphere with radius described by Eq. (5.2) and collapsing into a single point at time $T=0.25$.

Figure 2 shows the evolution of the sphere $\Omega_{n}$ obtained by the numerical approximation of problem (4.22), at different time instances; the evolution of the area $\left|\Omega_{n}\right|$ is reported in Figure 3, together with the evolution of the exact area $\left|\Omega_{t_{n}}\right|$ computed with Eq. (5.2). The sphere is represented by NURBS of degree $p=2$ and $C^{1}$-continuous a.e., for a total of 220 elements, yielding 384 DOFs $^{1}$. Time advancement is performed employing a BDF scheme of order $k=2$ with fixed

\footnotetext{
${ }^{1}$ The amount of DOFs reported corresponds to the size of the linear system solved at each time step; therefore, it takes into account for the constraints set to build the periodic basis functions and the fact that the solution is vector valued (the velocity $\mathbf{v} \in \mathbb{R}^{3}$ for each control point).
} 


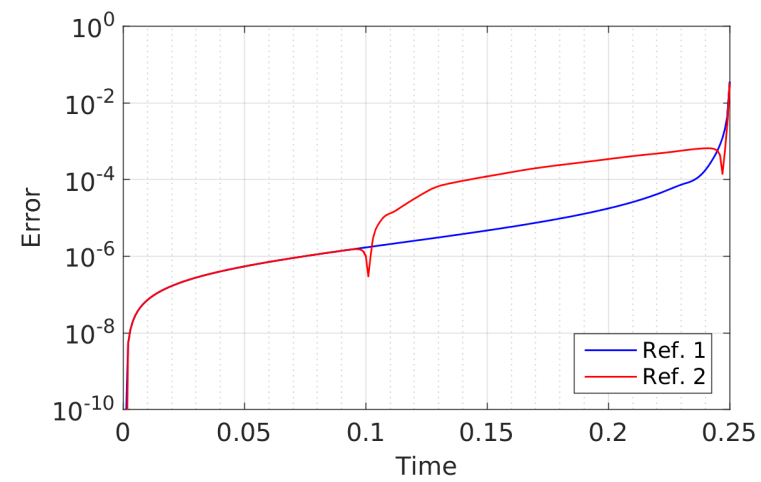

(a) $p=2, C^{1}$-continuous a.e., $3^{2}$ quad. pts.

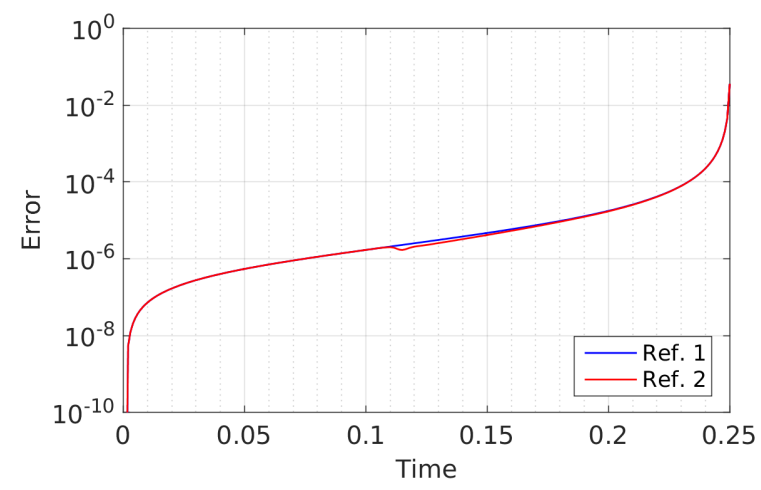

(c) $p=2, C^{1}$-continuous a.e., $7^{2}$ quad. pts.

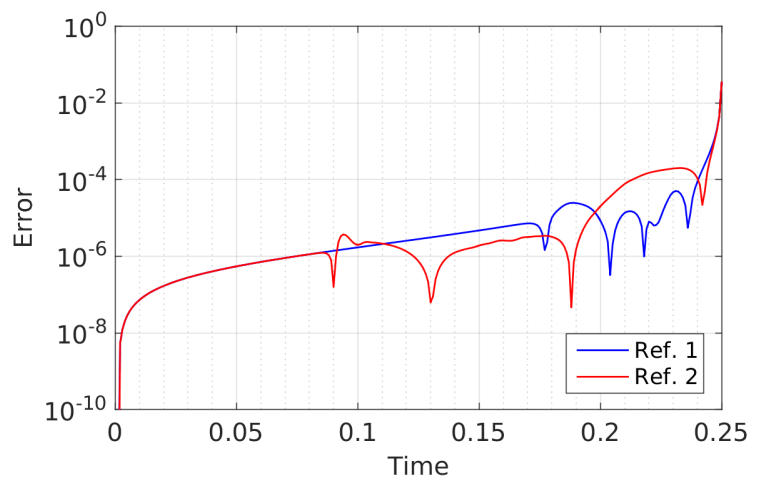

(b) $p=3, C^{2}$-continuous a.e., $4^{2}$ quad. pts.

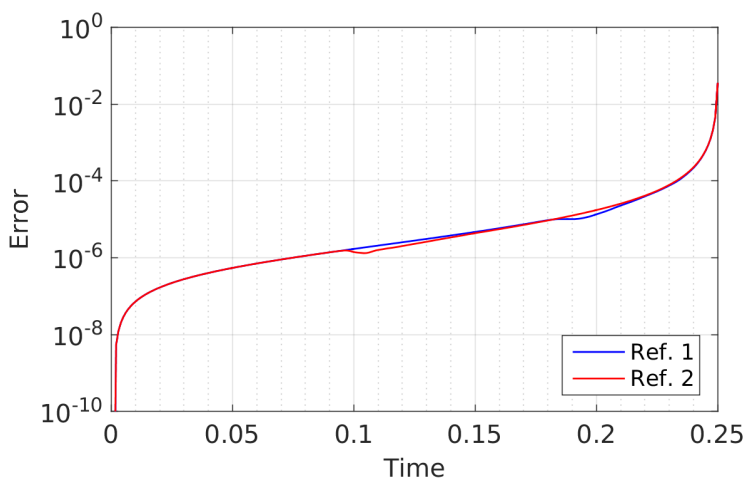

(d) $p=3, C^{2}$-continuous a.e., $7^{2}$ quad. pts.

Figure 4: Test 5.1.1. Mean curvature flow of a sphere. Evolution of the errors on the area err $_{n}$ vs time $t$ for meshes with different NURBS basis functions (ref. 1 with 384 DOFs and ref. 2 with 6,144 DOFs) and using $(p+1)^{2}$ (in (a) and (b)) and $7^{2}$ (in (c) and (d)) quadrature points per mesh element.

time step size $\Delta t=0.001$. The linear systems arising from the full discretization of the PDEs at each time step are solved by using the GMRES method with the ILUT preconditioner [52], with the stopping criterion being the relative residual (in Euclidean norm) below a tolerance of $10^{-9}$.

We report in Figure 4 the behavior of the errors on the numerically approximated area vs time, say $\operatorname{err}_{n}:=\left|\Omega_{t_{n}}-\Omega_{n}\right|$, obtained by solving problem (4.22) with NURBS of degree $p=2$ and 3 , which are $C^{p-1}$-continuous a.e., respectively. We compare the errors obtained using meshes of 220 and 2,380 elements for the $p=2, C^{1}$-continuous NURBS basis, while 275 and 2,555 elements for the $p=3, C^{2}$-continuous basis (yielding 384 and 6,144 DOF for both $p=2$ and 3). In particular, Figures $4 \mathrm{a}$ and $4 \mathrm{~b}$ show the errors obtained using the standard Gauss-Legendre quadrature rule with $(p+1)^{2}$ points per mesh element for the approximation of the integrals, while Figures $4 \mathrm{c}$ and $4 \mathrm{~d}$ show the errors obtained using $7^{2}$ quadrature points per element, thus with a significantly 


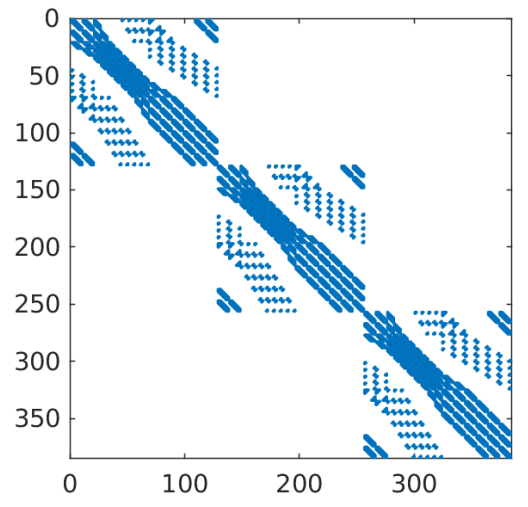

(a) $p=2, C^{1}$-continuous a.e.

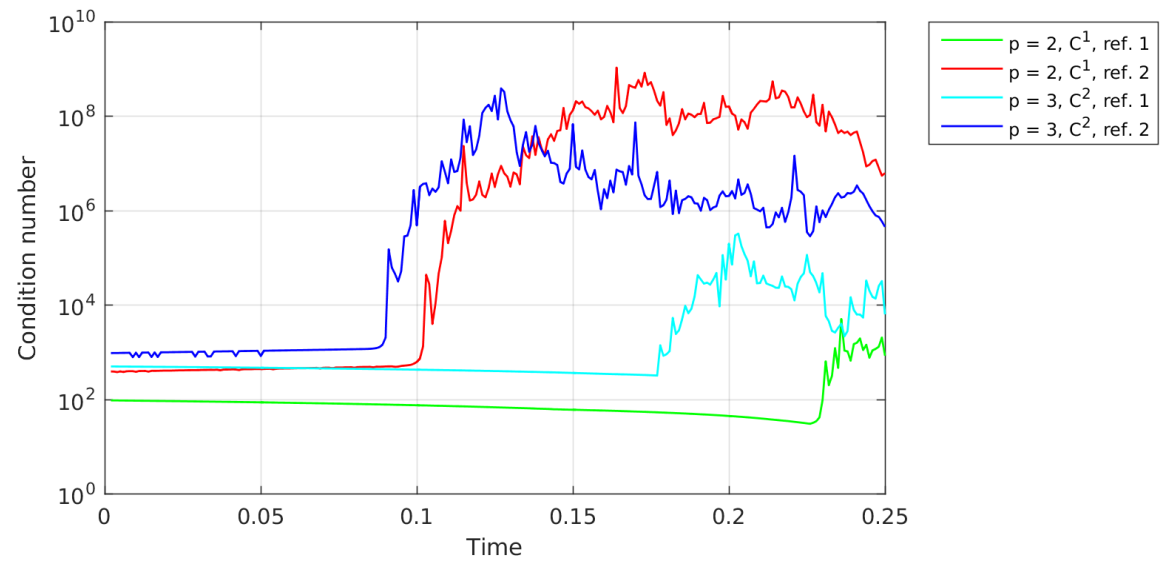

(c) Evolution of the condition number vs. time $t$

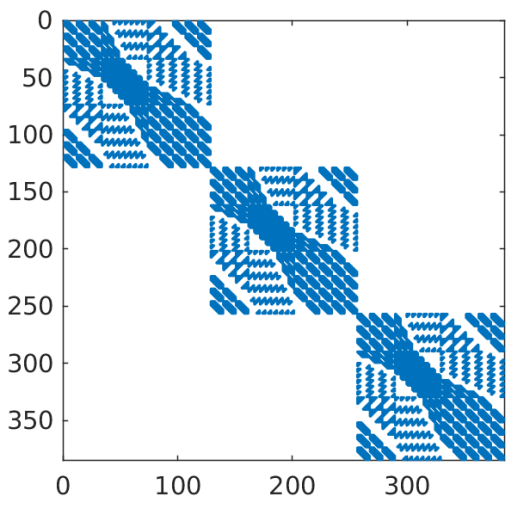

(b) $p=3, C^{2}$-continuous a.e.

Figure 5: Test 5.1.1. Mean curvature flow of a sphere. Sparsity patterns (a) and (b) and evolution of the condition number $\kappa(A)$ of the matrix associated to the full discrete problem (4.22) vs. time $t((\mathrm{c}))$, using NURBS basis functions of degrees $p=2$ and $3, C^{1}$ - and $C^{2}$-continuous a.e., respectively, and two refinement levels yielding 384 and 6,144 DOFs, respectively.

increased accuracy of the numerical integration. We observe that the errors are very small in all the cases, and only increase when the geometry tends to degenerate in a point, as expected from the exact solution of Eq. (5.2). In addition, a smoother behavior of the error $\operatorname{err}_{n}$ is observed when using a large number of quadrature nodes. Nevertheless, the errors remain very small, even for the standard Gauss-Legendre quadrature formulas with $(p+1)^{2}$ points typically used in IGA and employed in the rest of this work. We report in Figures 5a and 5b the sparsity patterns of the matrices $A$ arising from the full discrete problem (4.22) with NURBS of degree $p=2$ and 3 , respectively, with 384 DOFs in both the cases. In Figure 5c the evolutions of the condition number of the matrices associated to problem (4.22) at each time step are reported for the NURBS already 


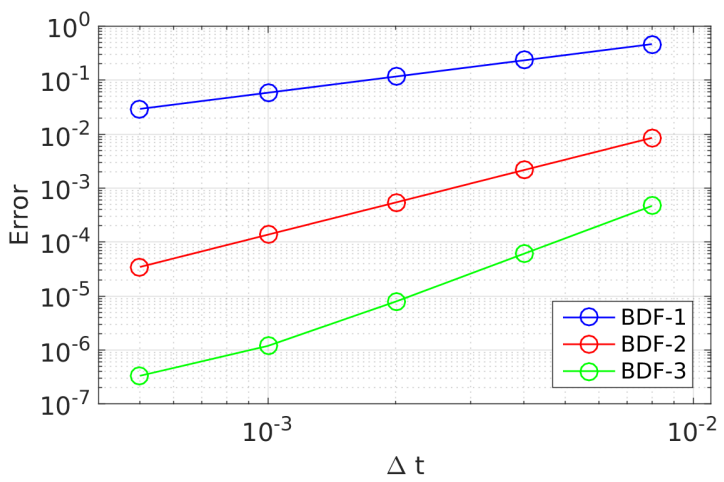

(a) $p=2, C^{1}$-continuous a.e.

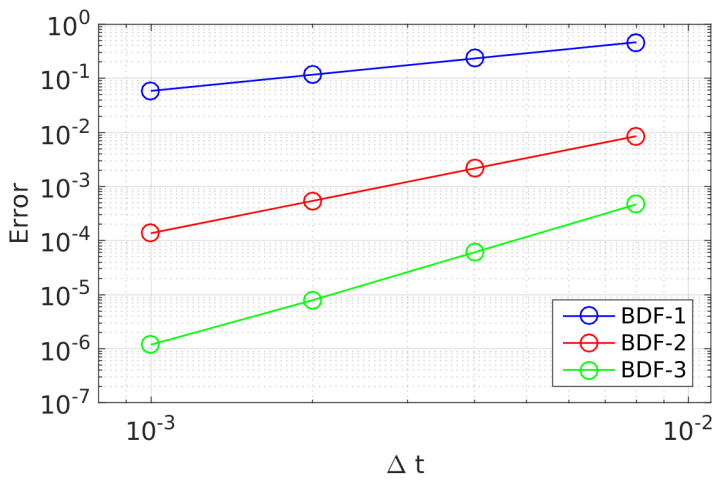

(c) $p=2, C^{0}$-continuous a.e.

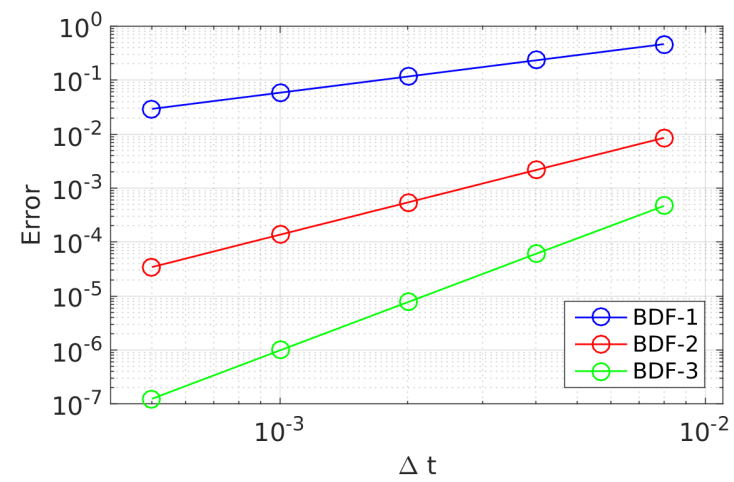

(b) $p=3, C^{2}$-continuous a.e.

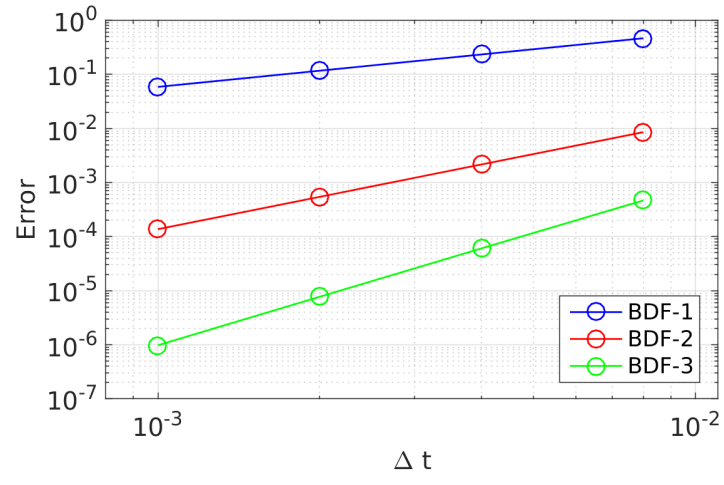

(d) $p=3, C^{0}$-continuous a.e.

Figure 6: Test 5.1.1. Mean curvature flow of a sphere. Absolute errors on the area at time $t_{\tilde{n}}=0.016$ err $r_{\tilde{n}}$ vs. $\Delta t$, for different BDF schemes (BDF of orders $k=1,2$, and 3) and NURBS basis functions ( $p=2$ and 3, which are $C^{0}$ - and $C^{p-1}$-continuous a.e.).

considered for the results in Figure 4; the condition number $\kappa(A)$ is actually a lower bound of the 1-norm condition number of the matrix $A$. The condition numbers $\kappa$ increase with the degree of the NURBS basis functions and when the mesh is refined. We remark that, for this specific problem, the NURBS mapping is singular at the poles of the sphere, which leads to high values of the condition number. Moreover, the sphere shrinks according to the mean curvature flow, and eventually degenerates in a point. Nevertheless, in the case under consideration, the condition numbers $\kappa(A)$ are never high enough to significantly interfere with the accuracy of the GMRES solver (for the chosen tolerance).

In order to compare the performance of the proposed scheme with BDFs of different orders with respect to the time step size $\Delta t$, simulations with BDFs of orders $k=1,2$, and 3 have been performed, for meshes composed by NURBS basis functions of degrees $p=2$ and 3 , which are $C^{0}$ - 
and $C^{p-1}$-continuous a.e. on the surface. Errors on the area with respect to the exact solution are reported in Figure 6 in logarithmic scale; the reported errors are computed as $\operatorname{err}_{\tilde{n}}:=\left|\Omega_{t_{\tilde{n}}}-\Omega_{\tilde{n}}\right|$ at fixed time $t_{\tilde{n}}=0.016$, with $\tilde{n}=\frac{t_{\tilde{n}}}{\Delta t}$. The BDF schemes are initialized with the corresponding numbers of exact time steps in order to bootstrap the time integration method correctly, such that order of convergence of $k$ is maintained for each BDF used. The meshes considered are built out of NURBS basis functions of degrees $p=2$ and 3, with 220 and 275 elements, respectively; for the degree $p=2$, meshes with basis functions $C^{1}$-continuous a.e. and $C^{0}$-continuous a.e. are considered, with 384 and 600 DOFs, respectively; for the degree $p=3$, meshes with basis functions $C^{2}$-continuous a.e. and $C^{0}$-continuous a.e. are considered, with 384 and 864 DOFs respectively. We remark that the errors corresponding to the spatial discretization are significantly small, even when approximating the problem with a low amount of mesh elements; this fact permits us to employ high order temporal discretizations and recover the full rate of convergence. Also, since the smooth $C^{p-1}$-continuous basis functions lead to a smaller number of DOFs than their $C^{0}$-continuous counterpart, the former generally lead to more efficient and accurate discretizations.

Test 5.1.2. Next, we study the evolution of a torus subject to mean curvature flow. We consider a family of toric surfaces $\Omega_{0}$ described by the relation:

$$
\left(R_{0}-\sqrt{x^{2}+y^{2}}\right)^{2}+z^{2}=r_{0}^{2}
$$

in a standard Cartesian coordinate system, where $R_{0}$ and $r_{0}$ are the torus' major and minor radii, respectively, being $R_{0}>0$ and $r_{0} \in\left(0, R_{0}\right)$, of the initial configuration corresponding to $\Omega_{0}$. Depending on the ratio between the two radii $R_{0} / r_{0}$, the torus is evolving either to collapse into a circle or to self-penetrate and merge into a disk. Figures 7 and 8 show the evolution of tori with $R_{0}=1, r_{0}=0.5$ and $R_{0}=1, r_{0}=0.7$, respectively, subject to mean curvature flow. The first torus has an aspect ratio $R_{0} / r_{0}$ such that it collapses into a circle, while the second one tends to merge into an ellipsoid; since we adopt a parametric representation of the geometry and we do not support topology changes, we let the geometry evolve until a self-intersection of the surface occurs. The evolution of the toric areas is plotted in Figures 9a and 9b, respectively. We consider NURBS with basis functions of degree $p=2$ and globally $C^{1}$-continuous, with 836 elements and 1,536 DOFs for both the cases; we used a BDF scheme of order $k=2$ for integration in time with time step size $\Delta t=0.001$.

Test 5.1.3. Now we consider the mean curvature flow of an open surface, in particular a cylindrical shell. We parametrize the cylinder by its radius $R_{0}$ and height $L_{0}$. The bottom and 

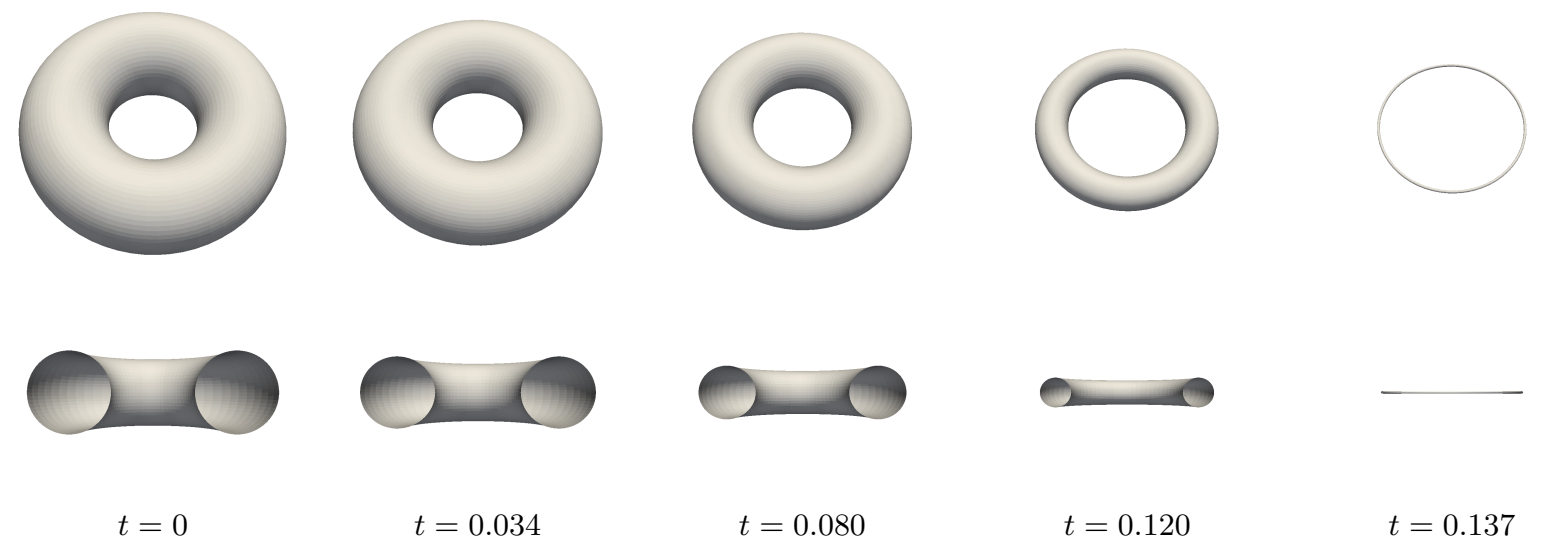

$t=0$

$t=0.034$

$t=0.080$

$t=0.137$

Figure 7: Test 5.1.2. Mean curvature flow of a torus with $R_{0}=1$ and $r_{0}=0.5$. Solution at different time instances.

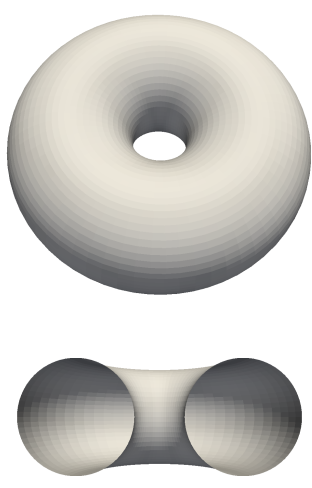

$t=0$
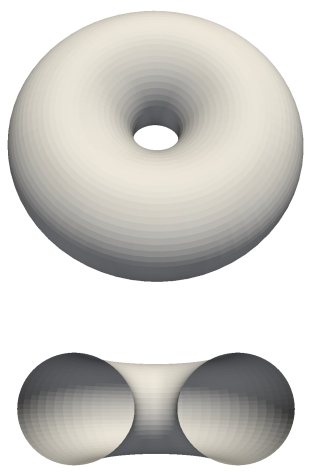

$t=0.024$
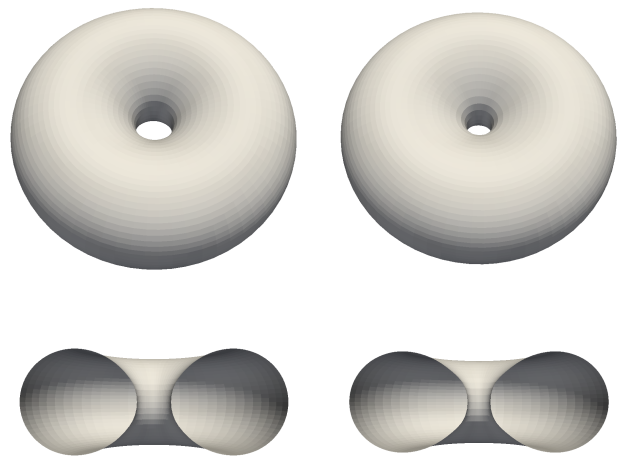

$t=0.048$

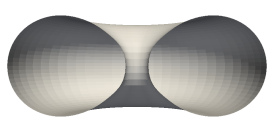

$t=0.072$
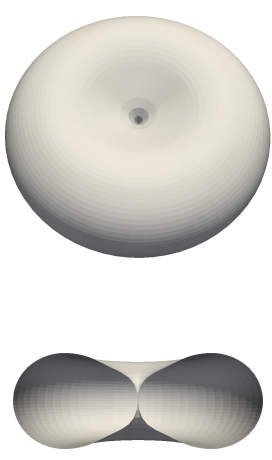

$t=0.095$

Figure 8: Test 5.1.2. Mean curvature flow of a torus with $R_{0}=1$ and $r_{0}=0.7$. Solution at different time instances.

top bases of the cylinder (two circles of radius $R_{0}$ ) are fixed (i.e. we set $\mathbf{x}_{h}=\mathbf{x}_{0, h}$ on $\partial \Omega$ ), while the lateral surface $\left(\Omega_{t}\right)$ is free to evolve according to the mean curvature flow. The geometry minimizing the area depends on the aspect ratio $L_{0} / R_{0}$ of the initial cylinder $\Omega_{0}$. In particular, the solution may either be discontinuous, consisting in two circles at the bases of the cylinder, and thus involving a topology change (known as Goldschmidt solution [62]), or exhibit a catenoid as local minimum, generated by rotating the catenary of equation $x=a \cosh \left(\frac{y}{a}\right)$ along the $y$-axis, with $a \in \mathbb{R}$ being solution of the nonlinear relation $\cosh \left(\frac{L_{0}}{2 a}\right)-\frac{R_{0}}{a}=0$. Such catenoid has area equal to:

$$
A_{\text {cat }}=\pi a^{2}\left[\sinh \left(\frac{L_{0}}{a}\right)+\frac{L_{0}}{a}\right] .
$$

Figures 10 and 11 show the evolution of two cylinders, the first with radius $R_{0}=1$ and height 


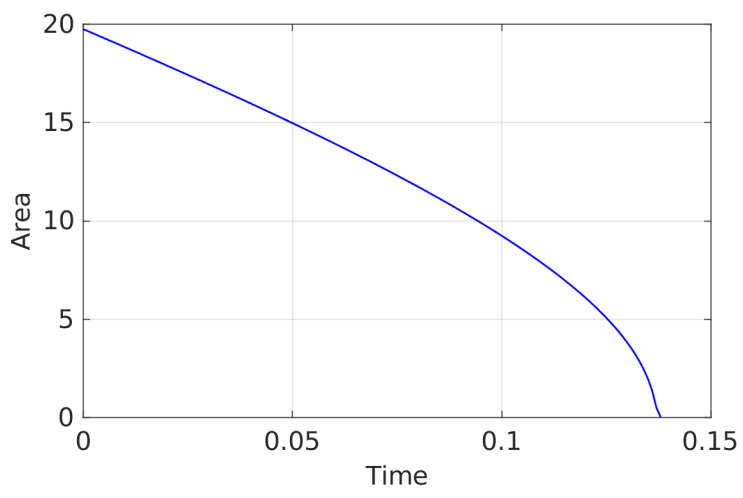

(a) Torus with $R=1$ and $r=0.5$

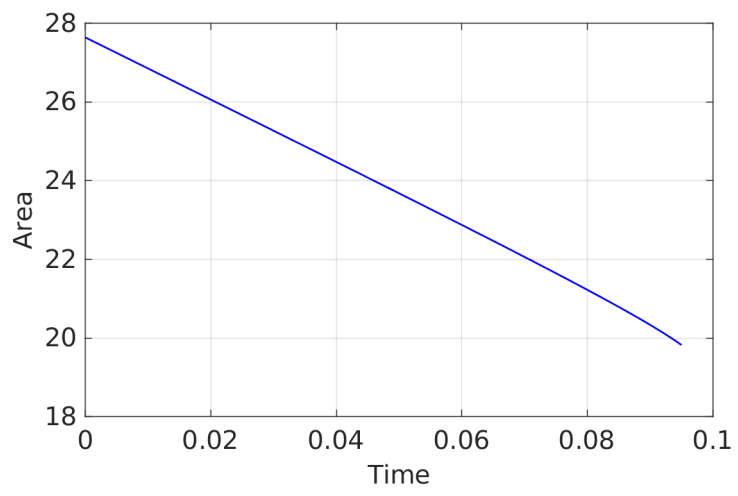

(b) Torus with $R=1$ and $r=0.7$

Figure 9: Test 5.1.2. Mean curvature flow of tori. Evolution of the approximated area $\left|\Omega_{n}\right|$ vs. time $t$ for a torus with $R_{0}=1$ and $r_{0}=0.5$ (a) and a torus with $R_{0}=1$ and $r_{0}=0.7$ (b); NURBS of degree $p=2$ and globally $C^{1}$-continuous with 836 mesh elements, yielding 1,536 DOFs, are used.

$L_{0}=1$, while the second one with $R_{0}=1$ and $L_{0}=2$. The meshes considered in the spatial approximation are both NURBS built of basis functions of degree $p=2$ and globally $C^{1}$-continuous, with 456 elements, for a total of 1,152 DOFs; time integration is performed employing a BDF scheme of order 2 , with time step size $\Delta t=0.001$. The evolutions of the areas $\left|\Omega_{t}\right|$ are plotted in Figures 12a and $12 \mathrm{~b}$, respectively. The first cylinder has aspect ratio $L_{0} / R_{0}=1$ such that a catenoid is a local minimum and the numerical solution effectively converges to such geometry. The second cylinder (for $L_{0} / R_{0}=2$ ) does not present a catenoid as local minimum, therefore the minimization process continues towards the solution with topology changes, which we stop when a singularity in the geometrical mapping is reached, as indicator of a topology change. In both the cases, we obtain accurate solutions even by employing spatial discretizations involving a small amounts of mesh elements and DOFs.

\subsection{Willmore flow}

We now consider the numerical approximation of the Willmore flow problem defined in Eq. (3.18) on closed surfaces using the numerical scheme (4.13) proposed in Section 4.1.4. For all the tests we set $\mu=1$ (see Eq. (3.17)).

Test 5.2.1. As initial geometry $\Omega_{0}$ we consider ellipsoids described by the following relation:

$$
\frac{x^{2}}{a_{0}^{2}}+\frac{y^{2}}{b_{0}^{2}}+\frac{z^{2}}{c_{0}^{2}}=1 \quad\{x, y, z\} \in \mathbb{R}^{3},
$$




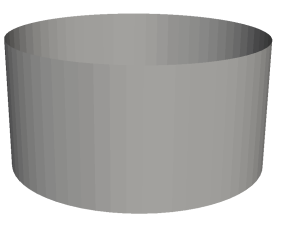

(a) $t=0$

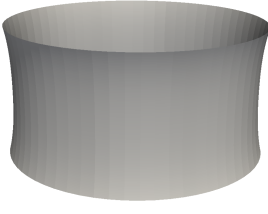

(b) $t=0.05$

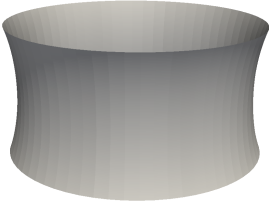

(c) $t=0.10$

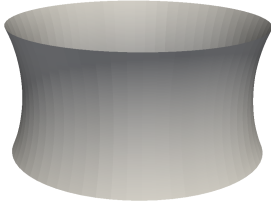

(d) $t=0.15$

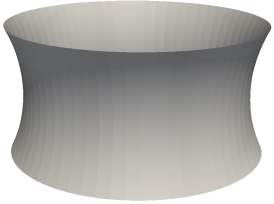

(e) $t=0.20$

Figure 10: Test 5.1.3. Mean curvature flow of a cylinder with $R_{0}=1$ and $L_{0}=1$. Solution at different time instances.

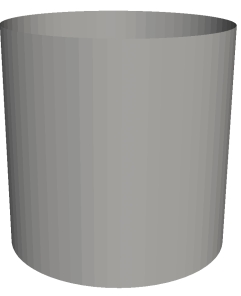

(a) $t=0$

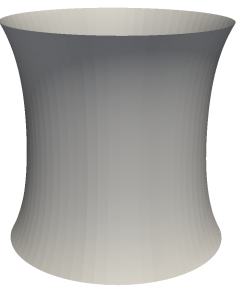

(b) $t=0.015$

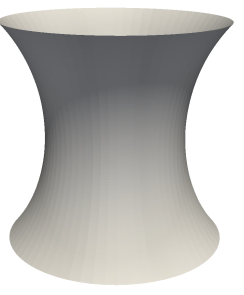

(c) $t=0.090$

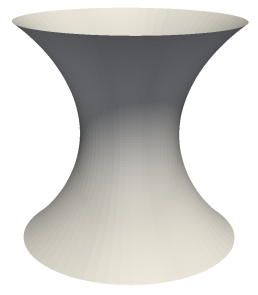

(d) $t=0.180$

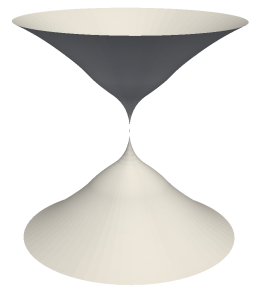

(e) $t=0.270$

Figure 11: Test 5.1.3. Mean curvature flow of a cylinder with $R_{0}=1$ and $L_{0}=2$. Solution at different time instances.

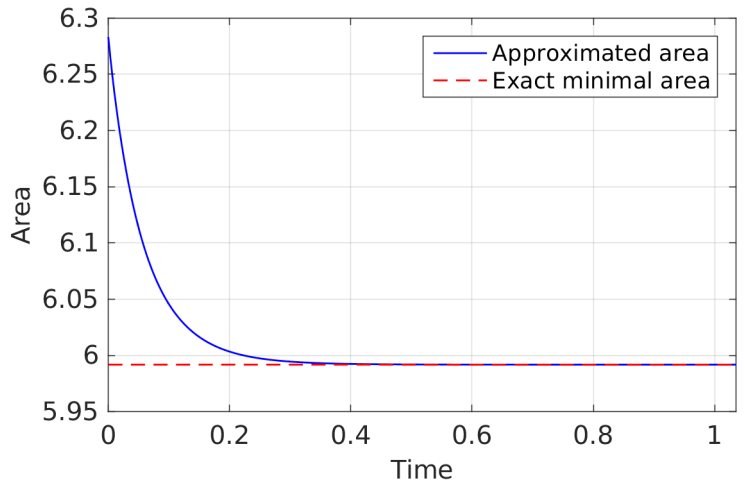

(a) Cylinder with $R_{0}=1$ and $L_{0}=1$

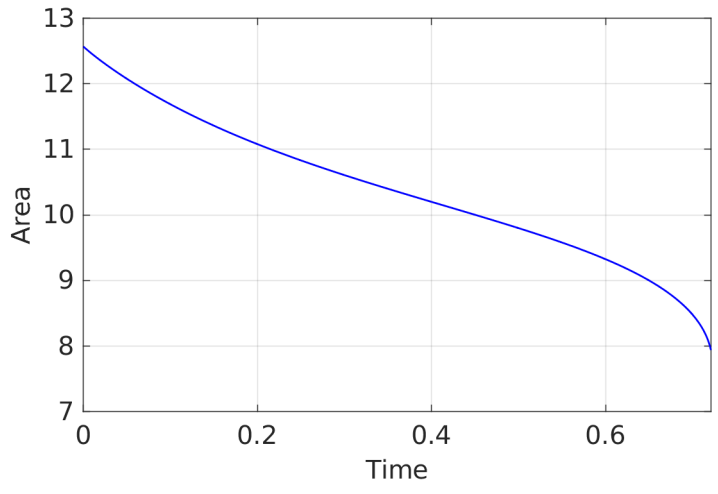

(b) Cylinder with $R_{0}=1$ and $L_{0}=2$

Figure 12: Test 5.1.3. Mean curvature flow of cylinders. Evolution of the approximated area $\left|\Omega_{n}\right|$ vs. time $t$ for a cylinder with $R_{0}=1$ and $L_{0}=1$ (a) and with $R_{0}=1$ and $L_{0}=2$ (b); NURBS of degree $p=2$ and globally $C^{1}$-continuous with 456 mesh elements, yielding 1,152 DOFs, are used. 

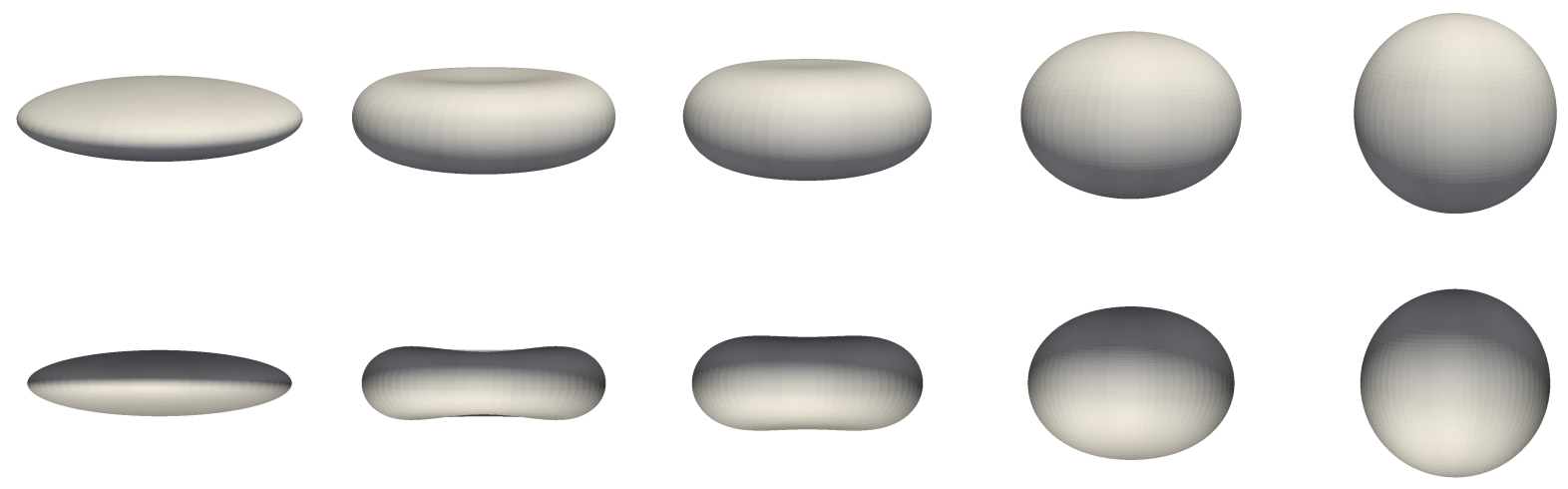

$t=0$

$t=0.49$

$t=1.22$

$t=3.66$

$t=12.21$

Figure 13: Test 5.2.1. Willmore flow of an ellipsoid with $a_{0}=4, b_{0}=4$ and $c_{0}=1$. Solution at different time instances.

where $a_{0}, b_{0}, c_{0} \in \mathbb{R}$ are positive constants denoting its aspect ratio. It is known that an ellipsoid should converge to a sphere under Willmore flow [31], which has Willmore energy $J_{W}$ equal to $8 \pi$. For our numerical test, we consider the approximation of the Willmore flow applied to an initial ellipsoid $\Omega_{0}$ with parameters $a_{0}=4, b_{0}=4$, and $c_{0}=1$. The geometry $\Omega_{0}$ is represented as a NURBS surface with basis functions of degrees $p=2$ and 3 , being $C^{1}$ - and $C^{2}$-continuous a.e., respectively, with two $h$-refinement levels for each degree. The considered meshes with NURBS of degree $p=2$ are made of 684 and 2,380 elements, respectively for the two refinement levels; the meshes with basis functions of degree $p=3$ are instead made of 779 and 2,555 elements, respectively. With respect to the two $h$-refinement levels, the total number of DOFs amounts to 2,048 and 8,192, independently of the degree $p$ of the NURBS basis functions ${ }^{2}$. Integration in time is performed employing the BDF scheme of order $k=2$ with a fixed time step size $\Delta t=0.01$. Figure 13 shows the solution obtained at different time steps, with the mesh composed of 779 elements. The evolution in time of the Willmore energy, together with the Willmore energy associated to a sphere (indicated as Exact final energy), is reported in Figure 14, together with the evolution in time of the area and the volume of the approximated geometry $\Omega_{n}$. We remark that problem (3.18) does not involve any constraint on the area and the volume of the surface, which are in principle free to evolve while the Willmore energy $J_{W}$ is being minimized; as a matter of fact, we notice that

\footnotetext{
${ }^{2}$ The number of DOFs accounts for both a vector valued unknown (the velocity $\mathbf{v}$ ) and a scalar unknown (the normal velocity $v$ ).
} 

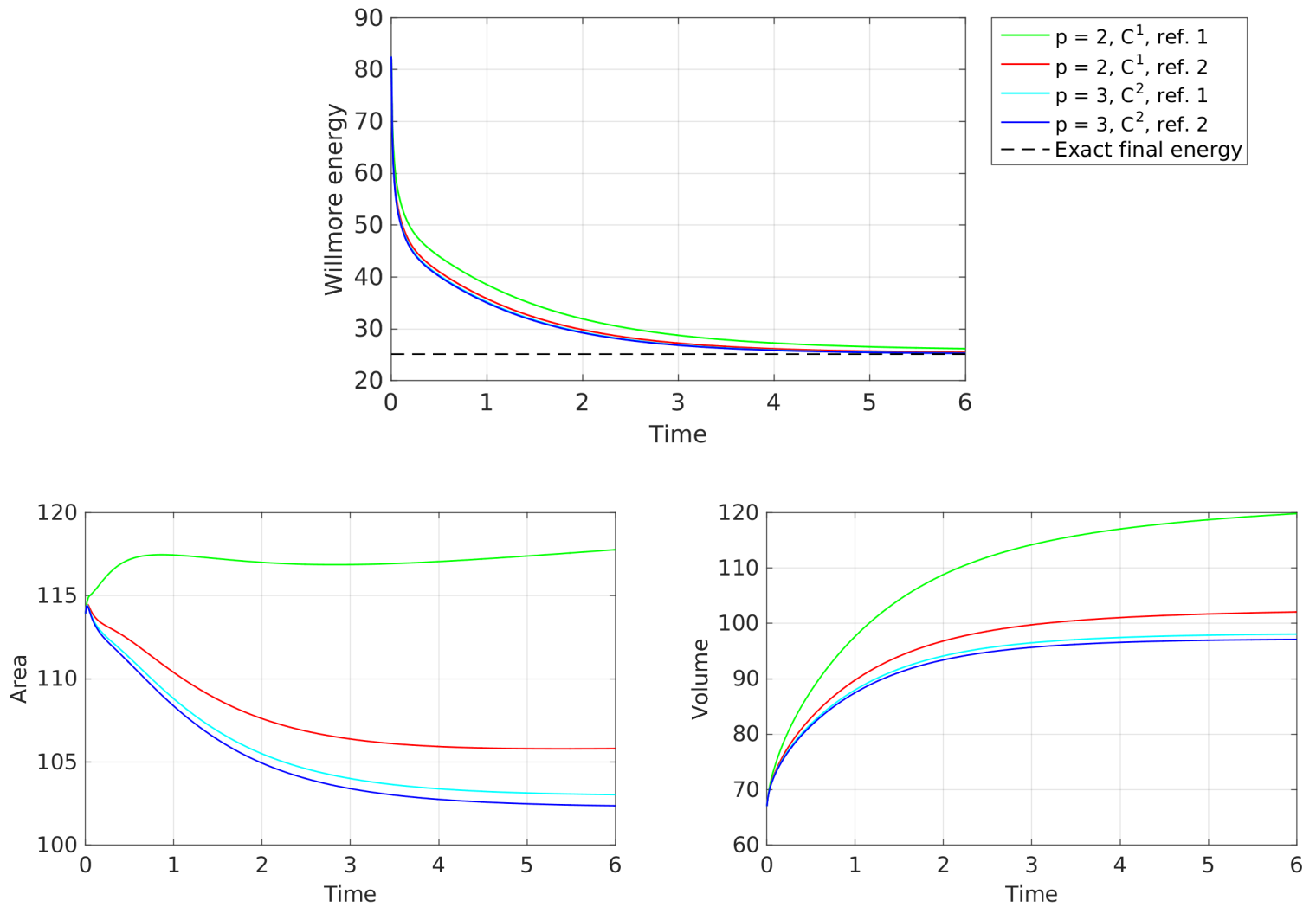

Figure 14: Test 5.2.1. Willmore flow of an ellipsoid with $a_{0}=4, b_{0}=4$ and $c_{0}=1$. Evolution of the Willmore energy $J_{W}$, area and volume vs. time $t$ (zoom) for NURBS of degrees $p=2$ and $C^{1}$-continuous a.e. with 684 and 2,380 elements, yielding 2,048 and 8,192 DOFs, respectively, and $p=3$ and $C^{2}$-continuous a.e. with 779 and 2,555 elements, again yielding 2,048 and 8,192 DOFs, respectively.

the evolutions of the area and the volume are sensitive to the discretization under consideration. By using as stopping criterion for the Willmore flow the difference between the Willmore energy at two consecutive time steps, which should be under the threshold $10^{-5}$, we obtain, with the coarsest mesh built of NURBS of degree $p=2$ a final error on the Willmore energy equal to 0.6496 $(2.585 \%)$; when refining the mesh, we obtain a significant reduction of such error, being equal to $0.1696(0.675 \%)$. Instead, using NURBS of degree $p=3$ yields better results, with errors equal to $0.0237(0.094 \%)$ and $0.0055(0.022 \%)$ for the first and second $h$-refinement levels, respectively. Finally, we report in Figures 15a and 15b the sparsity patterns of the matrices associated to the full discrete problem (4.26) with NURBS of degrees $p=2$ and 3, with 2,048 DOFs for both the cases. In Figure 15c the evolution of the condition number $\kappa(A)$ of the matrices involved in problem (4.26) is reported at each time step, for each NURBS considered for the results of Figure 14. The behavior of $\kappa(A)$ is similar to what experienced for Test 5.1.1, in the sense that, the higher degree of the 


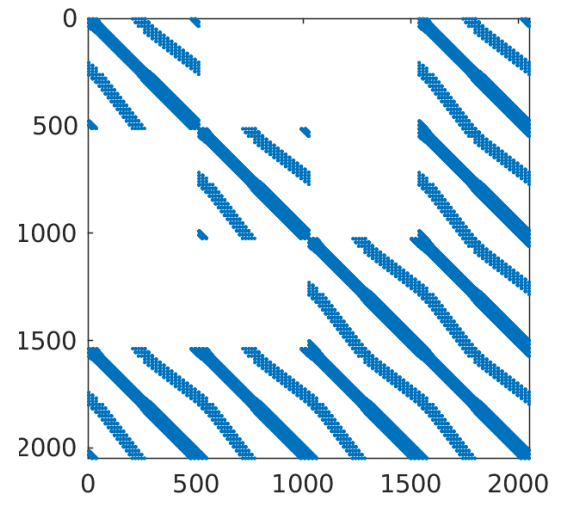

(a) $p=2, C^{1}$-continuous a.e.

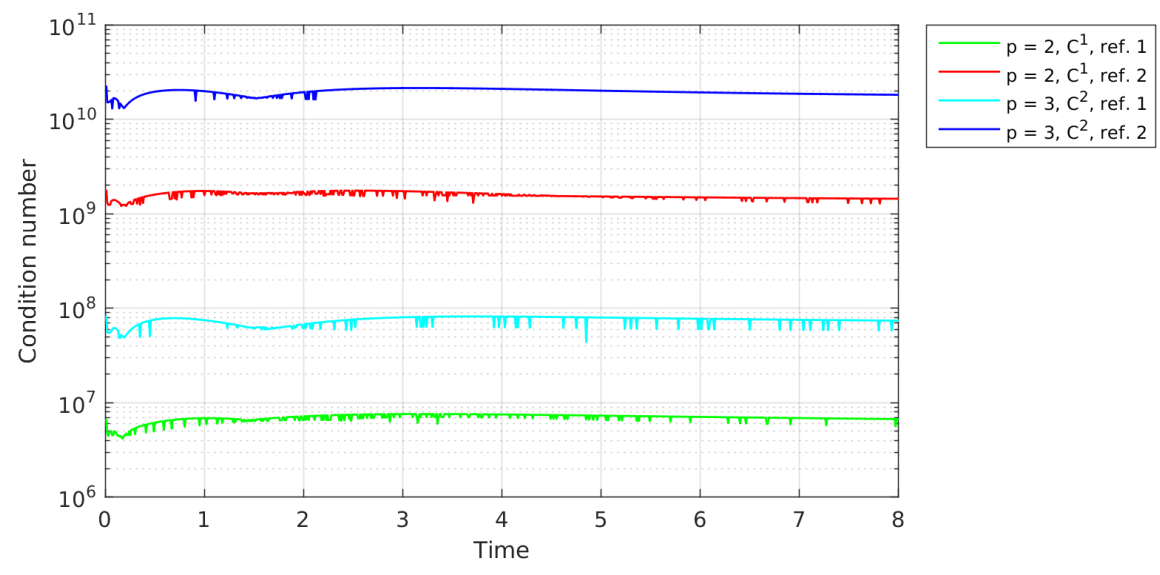

(c) Evolution of the condition number vs. time $t$

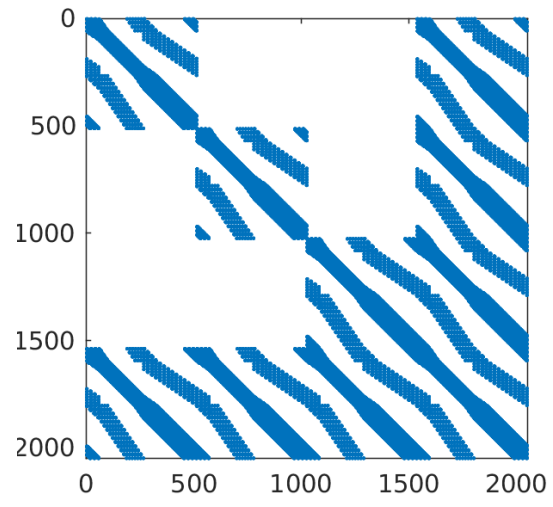

(b) $p=3, C^{2}$-continuous a.e.

Figure 15: Test 5.2.1. Willmore flow of an ellipsoid with $a_{0}=4, b_{0}=4$ and $c_{0}=1$. Sparsity patterns in (a) and (b) and evolution of the condition number $\kappa(A)$ of the matrix associated to the full discrete problem (4.26) vs. time $t((\mathrm{c}))$, using NURBS basis functions of degrees $p=2$ and $3, C^{1}$ - and $C^{2}$-continuous a.e., respectively, and two refinement levels yielding 2,048 and 8,192 DOFs, respectively, both for $p=2$ and $p=3$.

NURBS basis functions and the finer the mesh, the higher the condition number. With respect to Test 5.1.1, the condition number is generally higher, due to the high order derivatives involved in the Willmore flow problem.

Test 5.2.2. Now, we consider the numerical approximation of the Willmore flow of a torus, described by Eq. (5.3). In particular, Clifford tori, which are defined as having the ratio between the outer $R_{0}$ and inner $r_{0}$ radii equal to $R_{0} / r_{0}=\sqrt{2}$, are stationary geometries for the Willmore flow, with Willmore energy $J_{W}$ equal to $4 \pi^{2}$; tori with different aspect ratios tend to converge to the Clifford torus. We numerically simulate the Willmore flow of a initial torus $\Omega_{0}$ with $R_{0}=1$ 

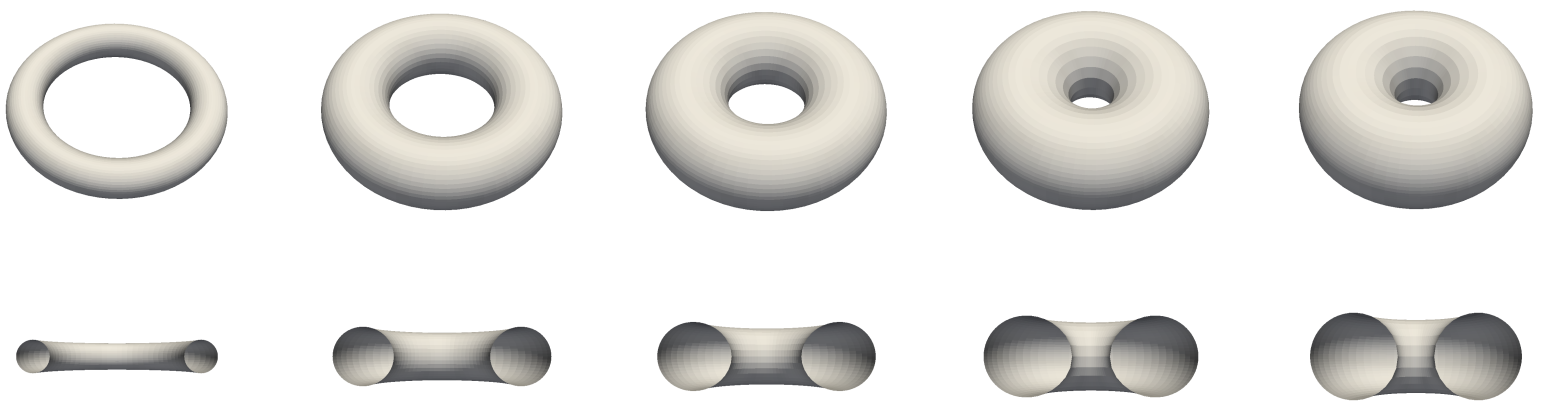

$t=0$

$t=0.003$

$t=0.010$

$t=0.030$

$t=0.100$

Figure 16: Test 5.2.2 Willmore flow of a torus with $R_{0}=1$ and $r_{0}=0.2$. Solution at different time instances.

and $r_{0}=0.2$ (i.e. for which $R_{0} / r_{0}=5$ ), represented as a NURBS surface with basis functions of degrees $p=2$ and 3 , being globally $C^{1}$ - and $C^{2}$-continuous, and two $h$-refinement levels. Solutions at different time steps are reported in Figure 16. Time discretization uses the BDF scheme of order $k=2$ and time step size $\Delta t=0.001$. We employed NURBS meshes built with 836 elements, yielding 2,048 DOFs, and 2,660 elements, yielding 8,192 DOFs, with NURBS basis functions of degree $p=2$ and globally $C^{1}$-continuous, and meshes with 1,025 elements, yielding 2,880 DOFs, and 2,993 elements, yielding 9,792 DOFs, with NURBS basis functions of degree $p=3$ and globally $C^{2}$-continuous. In Figure 17 the evolution of the Willmore energy is reported, together with the Willmore energy of the Clifford torus (indicated as Exact final energy). If we compare the final Willmore energy of the approximated solution with the Willmore energy of the Clifford torus we obtain the following errors with the above mentioned meshes, in order: 0.1146 (0.290\%), 0.1130 $(0.286 \%), 0.0114(0.029 \%)$, and $0.0010(0.003 \%)$. Therefore, the best compromise between accuracy and number of DOFs employed is obtained for NURBS basis functions of degree $p=3$ and globally $C^{2}$-continuous, which guarantee a good accuracy even with a small amount of DOFs. Finally, we report in Figures 18a and 18b the sparsity patterns of the matrices associated to the full discrete problem (4.26) with NURBS of degrees $p=2$ and 3, with 2,048 and 2,880 DOFs, respectively. We report in Figure 18c the evolution of the condition number $\kappa(A)$ at each time step, for each NURBS already considered in Figure 17. As usual, the condition numbers increase with the degree of the NURBS basis functions and with the refinement of the mesh, but, for each discretization, these follow the same overall behavior in time. With respect to Test 5.2.1, the condition number tends to be smaller, since the mapping does not present any singularity. 

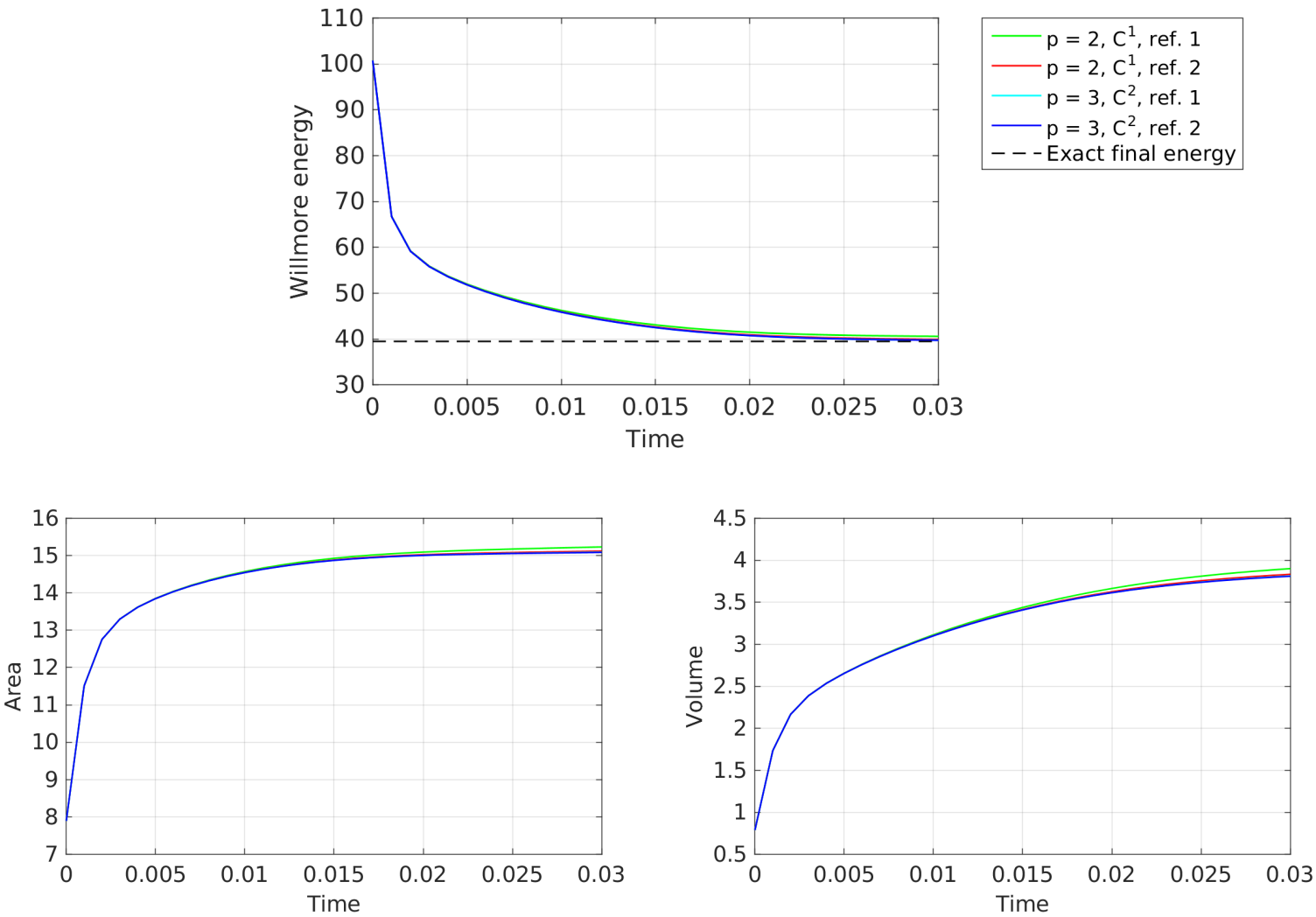

Figure 17: Test 5.2.2. Willmore flow of a torus with $R_{0}=1$ and $r_{0}=0.2$. Evolution of the Willmore energy $J_{W}$, area and volume vs. time $t$ (zoom) for meshes of two refinement levels built of NURBS of degrees $p=2$ (for 836 and 2,660 elements) and $p=3$ (for 1,025 and 2,993 elements), $C^{1}$ - and $C^{2}$-continuous a.e., respectively.

\section{Conclusions}

In this work, we considered the numerical approximation of geometric PDEs defined on surfaces by means of NURBS-based IGA in the framework of the Galerkin method. In particular, we focused on two problems: the mean curvature flow, leading to a nonlinear second order PDE deriving from the minimization of the area functional, and the Willmore flow, leading to a nonlinear fourth order PDE deriving from the minimization of the Willmore energy. We spatially approximated these problems with IGA, considering NURBS function spaces for representing both the geometry and the trial and test spaces for the spatial approximation of the PDEs, according with the isogeometric concept. In particular, we employed NURBS function spaces with basis functions featuring high order global continuity, necessary for the direct treatment of high order differential operators. For the time discretization, we considered high order BDF schemes, with extrapolation (of the same order of the BDF scheme) of geometric quantities from the solutions (the geometry) at the previous time steps. We reported results of the numerical approximation of the mean curvature flow on 


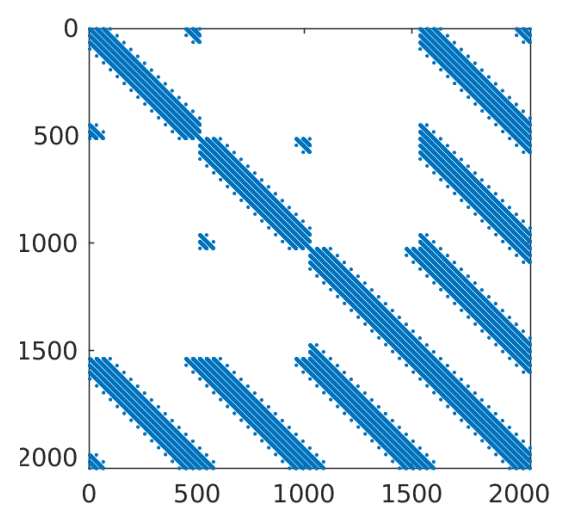

(a) $p=2, C^{1}$-continuous a.e.

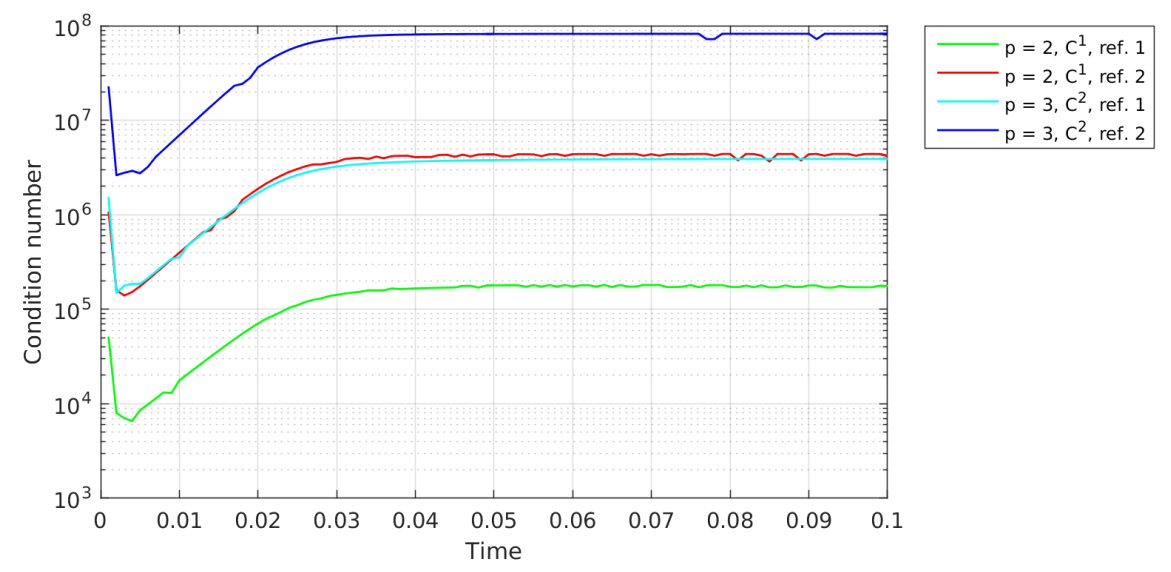

(c) Evolution of the condition number vs. time $t$

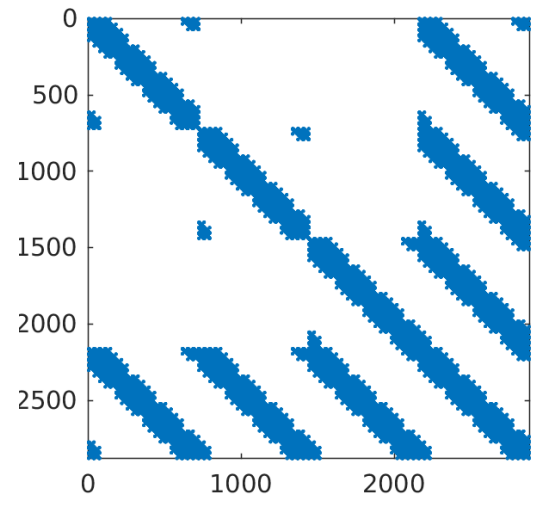

(b) $p=3, C^{2}$-continuous a.e.

Figure 18: Test 5.2.2. Willmore flow of a torus with $R_{0}=1$ and $r_{0}=0.2$. Sparsity patterns in (a) and (b) and evolution of the condition number $\kappa(A)$ of the matrix associated to the full discrete problem (4.26) vs. time $t((\mathrm{c}))$, using NURBS basis functions of degrees $p=2$ and $3, C^{1}$ - and $C^{2}$-continuous a.e., respectively, and two refinement levels yielding 2,048 and 8,192 DOFs for $p=2$, and 2,880 and 9,792 DOFs for $p=3$, respectively.

spheres, tori and cylinders, with error convergence analysis for BDF schemes of different orders and NURBS meshes with basis functions of different degrees and continuity, and of the Willmore flow on ellipsoids and tori, computed with different NURBS meshes. The numerical tests highlight that NURBS-based IGA is an efficient, accurate, and natural framework for the spatial approximation of geometric PDEs, including high order PDEs. The exact representation of the geometry, occurring even with a small amount of DOFs, together with the straightforward and accurate treatment of geometric quantities, proves to be extremely handful in this context. Moreover, the high degree of global continuity of the NURBS basis functions allows an efficient treatment of geometric PDEs, 
with high accuracy of the approximated solution using a smaller amount of DOFs. Furthermore, we reported the evolution of the condition numbers of the matrices associated with the full discrete problems, for which we highlighted that these may grow with the number of DOFs and the degree of the NURBS basis functions, especially in the presence of singularities of the geometrical mapping and the possible degeneration of the surface. Nevertheless, the approximate solution is very accurate already when few DOFs are involved in the discretization, i.e. for problems in which the condition number of the matrix is relatively small. As a matter of fact, the errors on the solution of the geometric PDEs associated to our numerical schemes are mainly driven by the time discretization errors, with the spatial ones basically annihilated by the IGA discretization.

\section{Acknowledgements}

The authors acknowledge the financial support of the Swiss National Science Foundation through the project "Isogeometric Analysis for Partial Differential Equations: surface models and optimization problems in Haemodynamics" (project $\sharp 147,033$ ).

\section{References}

[1] K. Deckelnick, G. Dziuk, C. M. Elliott, Computation of geometric partial differential equations and mean curvature flow, Acta Numerica 14 (2005) 139-232.

[2] J. W. Barrett, H. Garcke, R. Nürnberg, On stable parametric finite element methods for the Stefan problem and the Mullins-Sekerka problem with applications to dendritic growth, Journal of Computational Physics 229 (18) (2010) 6270-6299.

[3] J. W. Barrett, H. Garcke, R. Nürnberg, Numerical computations of faceted pattern formation in snow crystal growth, Physical Review E 86 (1) (2012) 011604.

[4] H. Garcke, Curvature driven interface evolution, Jahresbericht der Deutschen MathematikerVereinigung 115 (2) (2013) 63-100.

[5] A. Bonito, R. H. Nochetto, M. S. Pauletti, Parametric FEM for geometric biomembranes, Journal of Computational Physics 229 (9) (2010) 3171-3188.

[6] A. Bonito, R. H. Nochetto, M. S. Pauletti, Dynamics of biomembranes: effect of the bulk fluid, Mathematical Modelling of Natural Phenomena 6 (05) (2011) 25-43.

[7] M. S. Pauletti, Parametric AFEM for geometric evolution equation and coupled fluid-membrane interaction, Ph.D. thesis, University of Maryland (2008). 
[8] G. Aubert, P. Kornprobst, Mathematical problems in image processing (2002).

[9] T. Chan, L. Vese, Active contour and segmentation models using geometric PDEs for medical imaging, in: R. Malladi (Ed.), Geometric Methods in Bio-Medical Image Processing, Mathematics and Visualization, Springer Berlin Heidelberg, 2002, pp. 63-75.

[10] G. Sapiro, Geometric partial differential equations and image analysis, Cambridge University Press, 2006.

[11] Y. Duan, L. Yang, H. Qin, D. Samaras, Shape reconstruction from 3D and 2D data using PDE-based deformable surfaces, in: Computer Vision-ECCV 2004, Springer, 2004, pp. 238-251.

[12] H.-K. Zhao, S. Osher, R. Fedkiw, Fast surface reconstruction using the level set method, in: Variational and Level Set Methods in Computer Vision, 2001. Proceedings. IEEE Workshop on, IEEE, 2001, pp. 194-201.

[13] D. Liu, G. Xu, A general sixth order geometric partial differential equation and its application in surface modeling, Journal of Information and Computational Science 4 (1) (2007) 129-140.

[14] Q. Zhang, G. Xu, J. Sun, A general sixth order geometric flow and its applications in surface processing, in: Cyberworlds, 2007. CW'07. International Conference on, IEEE, 2007, pp. 447-456.

[15] U. Clarenz, U. Diewald, G. Dziuk, M. Rumpf, R. Rusu, A finite element method for surface restoration with smooth boundary conditions, Computer Aided Geometric Design 21 (5) (2004) 427-445.

[16] K. A. Brakke, The Motion of a Surface by its Mean Curvature, Princeton University Press, New Jersey, 1978.

[17] G. Huisken, Asymptotic behaviour for singularities of the mean curvature flow, Journal of Differential Geometry 31 (1) (1990) 285-299.

[18] G. Huisken, Flow by mean curvature of convex surfaces into spheres, Journal of Differential Geometry 20 (1984) 237-266.

[19] G. Huisken, Non-parametric mean-curvature evolution with boundary-conditions, Journal of Differential Equations 77 (2) (1989) 369-378.

[20] G. Dziuk, An algorithm for evolutionary surfaces, Numerische Mathematik 58 (1) (1990) 603-611.

[21] J. W. Barrett, H. Garcke, R. Nürnberg, On the parametric finite element approximation of evolving hypersurfaces in $\mathbb{R}^{3}$, Journal of Computational Physics 227 (9) (2008) 4281-4307.

[22] L. C. Evans, J. Spruck, Motion of level sets by mean curvature I, Journal of Differential Geometry 33 (3) (1991) 635-681. 
[23] D. L. Chopp, Computing minimal surfaces via level set curvature flow, Journal of Computational Physics 106 (1) (1993) 77-91.

[24] T. J. Willmore, Riemannian Geometry, Oxford University Press, 1996.

[25] W. Welch, A. Witkin, Free-form shape design using triangulated surfaces, in: Proceedings of the 21st annual conference on Computer Graphics and Interactive Techniques, ACM, 1994, pp. 247-256.

[26] P. B. Canham, The minimum energy of bending as a possible explanation of the biconcave shape of the human red blood cell, Journal of Theoretical Biology 26 (1) (1970) 61-81.

[27] W. Helfrich, Elastic properties of lipid bilayers: theory and possible experiments, Zeitschrift für Naturforschung. Teil C: Biochemie, Biophysik, Biologie, Virologie 28 (11) (1973) 693.

[28] U. Seifert, Configurations of fluid membranes and vesicles, Advances in physics 46 (1) (1997) 13-137.

[29] E. Kuwert, R. Schatzle, Gradient flow for the Willmore functional, Communications in Analysis and Geometry 10 (2) (2002) 307-339.

[30] E. Kuwert, R. Schätzle, The Willmore flow with small initial energy, Journal of Differential Geometry 57 (3) (2001) 409-441.

[31] E. Kuwert, R. Schätzle, Removability of point singularities of Willmore surfaces, Annals of Mathematics 160 (2004) 315-357.

[32] G. Simonett, The Willmore flow near spheres, Differential and Integral Equations 14 (8) (2001) 10051014.

[33] G. Francis, J. M. Sullivan, R. B. Kusner, K. A. Brakke, C. Hartman, G. Chappell, The minimax sphere eversion, in: Visualization and Mathematics, Springer, 1997, pp. 3-20.

[34] U. F. Mayer, G. Simonett, A numerical scheme for axisymmetric solutions of curvature-driven free boundary problems, with applications to the Willmore flow, Interfaces and Free Boundaries 4 (1) (2002) 89-109.

[35] R. E. Rusu, An algorithm for the elastic flow of surfaces, Interfaces and Free Boundaries 7 (3) (2005) 229-239.

[36] G. Dziuk, Computational parametric Willmore flow, Numerische Mathematik 111 (1) (2008) 55-80.

[37] J. W. Barrett, H. Garcke, R. Nürnberg, Parametric approximation of Willmore flow and related geometric evolution equations, SIAM Journal on Scientific Computing 31 (1) (2008) 225-253. 
[38] M. Droske, M. Rumpf, A level set formulation for Willmore flow, Interfaces and Free Boundaries 6 (3) (2004) 361-378.

[39] G. Dziuk, E. Kuwert, R. Schatzle, Evolution of elastic curves in $\mathbb{R}^{n}$ : Existence and computation, SIAM Journal on Mathematical Analysis 33 (5) (2002) 1228-1245.

[40] J. W. Barrett, H. Garcke, R. Nürnberg, A parametric finite element method for fourth order geometric evolution equations, Journal of Computational Physics 222 (1) (2007) 441-467.

[41] G. Dziuk, C. M. Elliott, Finite element methods for surface PDEs, Acta Numerica 22 (2013) 289-396.

[42] L. A. Piegl, W. Tiller, The NURBS Book, Springer-Verlag, Berlin, 1997.

[43] J. A. Cottrell, T. J. R. Hughes, Y. Bazilevs, Isogeometric Analysis: Toward Integration of CAD and FEA, John Wiley \& Sons, 2009.

[44] T. J. R. Hughes, J. A. Cottrell, Y. Bazilevs, Isogeometric Analysis: CAD, finite elements, NURBS, exact geometry and mesh refinement, Computer Methods in Applied Mechanics and Engineering 194 (39) (2005) 4135-4195.

[45] A. Bartezzaghi, L. Dedè, A. Quarteroni, Isogeometric Analysis of high order partial differential equations on surfaces, Computer Methods in Applied Mechanics and Engineering 295 (2015) 446-469.

[46] J. A. Cottrell, T. J. R. Hughes, A. Reali, Studies of refinement and continuity in isogeometric structural analysis, Computer Methods in Applied Mechanics and Engineering 196 (41) (2007) 4160-4183.

[47] L. Dedè, C. Jäggli, A. Quarteroni, Isogeometric numerical dispersion analysis for two-dimensional elastic wave propagation, Computer Methods in Applied Mechanics and Engineering 284 (2015) 320-348.

[48] T. J. R. Hughes, A. Reali, G. Sangalli, Duality and unified analysis of discrete approximations in structural dynamics and wave propagation: comparison of p-method finite elements with k-method NURBS, Computer Methods in Applied Mechanics and Engineering 197 (49) (2008) 4104-4124.

[49] J. Liu, L. Dedè, J. A. Evans, M. J. Borden, T. J. R. Hughes, Isogeometric Analysis of the advective Cahn-Hilliard equation: spinodal decomposition under shear flow, Journal of Computational Physics 242 (2013) 321-350.

[50] A. Tagliabue, L. Dedè, A. Quarteroni, Isogeometric Analysis and error estimates for high order partial differential equations in fluid dynamics, Computers \& Fluids 102 (2014) 277-303.

[51] L. Dedè, A. Quarteroni, Isogeometric Analysis for second order Partial Differential Equations on surfaces, Computer Methods in Applied Mechanics and Engineering 284 (2015) 807-834. 
[52] A. Quarteroni, R. Sacco, F. Saleri, Numerical Mathematics, Springer-Verlag, Berlin and Heidelberg, 2007.

[53] P. Gervasio, F. Saleri, A. Veneziani, Algebraic fractional-step schemes with spectral methods for the incompressible Navier-Stokes equations, Journal of Computational Physics 214 (1) (2006) 347-365.

[54] M. Berger, A Panoramic View of Riemannian Geometry, Springer-Verlag, Berlin and Heidelberg, 2003.

[55] A. Bonito, R. H. Nochetto, M. S. Pauletti, Geometrically consistent mesh modification, SIAM Journal on Numerical Analysis 48 (5) (2010) 1877-1899.

[56] M. C. Delfour, J. P. Zolésio, Shapes and Geometries: Metrics, Analysis, Differential Calculus, and Optimization, SIAM, Philadelphia (PA), 2011.

[57] L. Ambrosio, N. Gigli, G. Savare, Gradient Flows in Metric Spaces and in the Space of Probability Measures, Birkhäuser Basel, 2008.

[58] P. C. Fife, Models for phase separation and their Mathematics, Electronic Journal of Differential Equations 2000 (48) (2000) 1-26.

[59] A. Polden, Curves and surfaces of least total curvature and fourth-order flows, Ph.D. thesis, Mathematisches Institut, Universität Tübingen (1996).

[60] D. Forti, L. Dedè, Semi-implicit BDF time discretization of the Navier-Stokes equations with VMS-LES modeling in a High Performance Computing framework, Computers \& Fluids 117 (2015) 168-182.

[61] G. S. Rao, Numerical Analysis, New Age International Publishers, New Delhi, 2009.

[62] E. W. Weisstein, Minimal surface of revolution. From MathWorld-A Wolfram Web Resource. URL http://mathworld.wolfram.com/MinimalSurfaceofRevolution.html 